\title{
The effect of angle of attack on flow-induced vibration of low-side-ratio rectangular cylinders
}

\author{
Tommaso Massai ${ }^{\text {a,* }}$, Jisheng Zhao ${ }^{\text {b }}$, David Lo Jacono ${ }^{c}$, Gianni Bartoli ${ }^{a}$, \\ John Sheridan ${ }^{b}$ \\ ${ }^{\text {a } I n t e r-U n i v e r s i t y ~ R e s e a r c h ~ C e n t r e ~ o n ~ B u i l d i n g ~ A e r o d y n a m i c s ~ a n d ~ W i n d ~ E n g i n e e r i n g ~(C R I A C I V), ~ C i v i l ~ a n d ~ E n v i r o n m e n t a l ~ E n g i n e e r i n g ~}$ \\ Department (DICeA), Florence University, via S. Marta, 50139 Florence, Italy \\ b Fluids Laboratory for Aeronautical and Industrial Research (FLAIR), Department of Mechanical and Aerospace Engineering, Monash \\ University, Melbourne, Victoria 3800, Australia \\ ' Institut de Mécanique des Fluides de Toulouse (IMFT), Université de Toulouse, CNRS, Toulouse, France
}

\section{A R T I C L E I N F O}

\section{Article history:}

Received 26 September 2017

Received in revised form 13 April 2018

Accepted 27 July 2018

\section{Keywords:}

FIV

VIV

Galloping

Sharp-edged rectangular bodies

VIV-galloping interference

\begin{abstract}
A B S T R A C T
This study investigates the effect of angle of attack on flow-induced vibrations (FIVs) of sharp-edged rectangular cylinders. In particular, the effect of the afterbody of a rectangular cross-section with low mass ratio and the side ratio ranging from 0.67 to 1.5 is analysed by changing the angle of attack with respect to the oncoming free-stream. As already shown for a different side ratio's rectangle, namely a square section (see Nemes et al., 2012), the angle of attack variation can make the flow-induced amplitude response switch between vortex-induced vibration (VIV) and galloping. Some considerations with respect to the interaction between the two phenomena typical of FIV, VIV and galloping, are also given for the side ratios of 1.5 and 0.67 . The amplitude and frequency responses are carefully analysed, comparing the results with those of a square section of comparable mass ratio. The results showed a marked effect of the after-body, even for slight increments of the angle of attack. This can result in different amplitude response curves, as classified by the features of the response. In addition, the influence of interacting higher harmonics components on the amplitude response is also shown and discussed.
\end{abstract}

(C) 2018 Elsevier Ltd. All rights reserved.

\section{Introduction}

This study examines the influence of the angle of attack variation on the flow-induced vibration (FIV) response of sharpedged rectangular cylinders. The cross-section investigated has a side ratio $(S R=B / D)$, defined as the ratio between the body width $(B)$ and depth facing the fluid flow $(D)$, of 1.5 in the zero angle of attack $\left(\alpha=0^{\circ}\right)$ configuration. The body is subjected to a free-stream water flow perpendicular to its long axis, and the cylinder is constrained to oscillate only transversely to the free stream. Generally, sharp-edged bluff bodies can be subjected to different forms of aeroelastic phenomena, such as flutter, galloping and vortex-induced vibration (VIV). For the $S R$ range investigated here two phenomena have been found to occur, VIV and galloping. If galloping can be referred to as a divergent (not self-limited) aerodynamic instability, generally occurring in a higher range of reduced velocities, one can identify VIV as a self-limited phenomenon occurring in a bounded lower velocity range. It occurs when an elastic or elastically-mounted bluff body is immersed in a moving fluid and the occurrence of a fluctuating pressure distribution on the body may induce a vibrational response at certain velocities or

\footnotetext{
* Corresponding author.

E-mail addresses: tommaso.massai@unifi.it (T. Massai), jisheng.zhao@monash.edu (J. Zhao), david.lojacono@imft.fr (D.L. Jacono), gianni.bartoli@unifi.it (G. Bartoli), john.sheridan@monash.edu (J. Sheridan).
} 
natural frequencies. Given that galloping is caused by the aerodynamic forces induced by the transverse motion of the body, there can be no instability without an initial displacement condition.

Consequently, a body's motion implies a change of angle of attack, see for example Parkinson and Smith (1964) and as illustrated in Fig. 1. Thus, galloping is categorized as a type of movement-induced excitation (MIE), whereas VIV is categorized as a type of instability-induced excitation (IIE), given it is associated with the flow instability involving local flow oscillations (see Naudascher and Rockwell, 2005). These two phenomena will be discussed here and their mutual interference investigated. From here on they are referred as interaction VIV-galloping or simply interaction.

Vortex-induced vibration has been extensively investigated firstly on circular cylinders (e.g. Feng, 1968; Sarpkaya, 1979; Bearman, 1984; Sarpkaya, 2004), and then other body shapes (Bearman and Davies, 1977; Bearman and Currie, 1979; Bearman and Obasaju, 1982; Zhao et al., 2018a). In addition to the full understanding of the vortex shedding physics (Gerrard, 1966; Bearman, 1967; Perry et al., 1982) there has also been considerable interest in conceiving strategies for the suppression of transverse vibrations, caused by the non-linear resonance between the frequency of vortex shedding $\left(f_{\mathrm{vs}}\right)$, occurring for any body with an appreciable afterbody, and its natural (structural) frequency of oscillation $\left(f_{n}\right)$. These studies were primarily conducted on circular section bodies because this means any consideration involving the orientation of the flow (or attack angle, $\alpha$ ) can be neglected. As a result, researchers can focus mainly on the role played by the Reynolds number, $(R e)$, in affecting other key features of the phenomenon, such as wake turbulence transitions, position of the separation points, lift and drag crisis, switching behaviour of the Strouhal number $(S t)$ in the super-critical range.

Apart from the practical engineering applications in civil and ocean engineering (light poles, power line cables, submerged structures e.g. offshore platform pillars, risers, wind turbine towers, mooring lines and spars), the circular section is taken as the canonical section for studying the singular effect of vortex shedding, as other forms of divergent instabilities (galloping or flutter) cannot occur, given the aerodynamic stability of the section. Conversely, for sharp-edged bluff bodies the separation points are fixed at the section's up-stream leading edge. In fact, the near-wake vortex structure presents a negligible Redependence, without changing flow regimes increasing its velocity. In contrast, it has been found that for the circular cylinder vortex shedding regimes depend on incoming flow velocity (Roshko, 1954; Bloor, 1964).

Galloping is an aerodynamic instability of slender non-axisymmetric structures caused by a self-excitation when the aerodynamic damping becomes negative. When a cross-section is aerodynamically unstable, as it is in this case, where there is a lack of axial symmetry, a characteristic of rectangular sections. According to the Glauert-Den Hartog incipient stability criterion (stating the instability of a system in case Eq. (1) is verified) (Glauert, 1919; Den Hartog, 1932), small-amplitude vibrations generate forces that increase in amplitude to large values as the flow velocity is increased.

$$
\left.\frac{d C_{\mathrm{L}}}{d \alpha}\right|_{\alpha_{0}}+C_{\mathrm{D}}\left(\alpha_{0}\right)<0
$$

where $C_{\mathrm{L}}$ and $C_{\mathrm{D}}$ are the lift and drag aerodynamic force coefficients defined in Fig. 1, while $\alpha_{0}$ indicated the angle of attack at rest. To-date the only suitable theory to predict transverse 1-DoF galloping oscillations onset velocity and the post-critical regime is the quasi-steady theory (QS), which has been successfully applied by Parkinson and Smith (1964). Once the galloping onset velocity (Eq. (4)), which is proportional to the mass and damping ratio, is exceeded the system manifests itself as limit-cycle harmonic oscillations.

The dynamics of an elastically mounted body constrained to oscillate across the stream depends on the mass of the oscillating body, $m$, the mechanical damping $c$ and the system elastic stiffness $k$ (both assumed constant here), the fluid density $\rho$, the kinematic viscosity $v$, and the free-stream velocity $U$. It is defined by the equation

$$
m \ddot{y}+c \dot{y}+k y=F_{y}(t),
$$

where $F_{y}(t)$ represents the forcing imposed on the cylinder by the fluid. This leads to the key non-dimensional parameters of the system typically used for FIV: the mass ratio, $m^{*}=m / m_{d}=m / \rho V$, where $m_{d}$ is the mass of the fluid displaced by the body, and $V=B D L$ the immersed body volume; and the damping ratio of the system in water,

$$
\zeta_{\mathrm{w}}=c /\left(2 \sqrt{k\left(m+m_{A}\right)}\right),
$$

where $m_{A}$ is the added mass. The added mass can be estimated from potential flow or measured directly through its influence on the natural frequency of the body in quiescent fluid. The reduced velocity is defined by $U^{*}=U /\left(f_{\mathrm{n}} D\right)$, where $f_{\mathrm{n}}$ is the natural frequency of the freely oscillating body in quiescent fluid and $D$ is the cylinder's characteristic transversal dimension. In his pioneering paper on the VIV of a circular cylinder in air flow, Feng (1968) showed that a resonance condition can exist when the frequency of shedding, $f_{\mathrm{vs}}$, and that of the body oscillation, $f_{o s c}$, are synchronized when close to $f_{\mathrm{n}}$. The maximum amplitude response, $A_{\max }^{*}=\max (A / D)$, occurs in this lock-in region of $f_{\mathrm{vs}} \approx f \approx f_{\mathrm{n}}$, i.e. after the reduced velocity increases above, $U_{r}^{*}=1 / S t$, where $S t=f_{\mathrm{vs}} D / U$ is the dimensionless shedding frequency of a fixed cylinder.

According to $Q S$-theory the galloping onset reduced velocity $\left(U_{g}^{*}\right)$ is given, in a different non-dimensional form to that reported in Eq. 19 of Parkinson and Smith (1964), as a function of damping $(\zeta)$, geometry of the cross-section $(B, D)$, fluid $\operatorname{density}(\rho)$, mass ratio $\left(m^{*}\right)$ and slope of the lateral force coefficient around the zero angle of attack, that is $A_{1}=\left.\left(d C_{\mathrm{Fy}} / d \alpha\right)\right|_{0^{\circ}}$.

$$
U_{g}^{*}=2 \pi \frac{2 \zeta}{n A_{1}}=\frac{4 m \zeta}{\rho D^{2} L A_{1}}=\frac{S c}{A_{1} \pi}=\frac{4 \zeta m^{*}}{A_{1}} \frac{B}{D},
$$




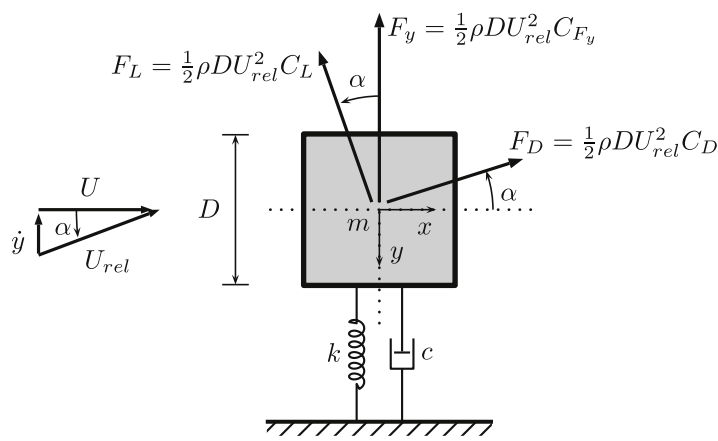

Fig. 1. Evaluation of the transverse force on a vibrating rectangular cylinder with the $Q S$ approach. $U_{\text {rel }}$ denotes the apparent flow velocity due to the body displacement velocity. Axes orientation $(x, y)$ are indicated, while $F_{L}, F_{D}, k$ and $c$ represent respectively the lift and drag aerodynamic forces on the body, and the mechanical stiffness and damping of the system.

where the Scruton number is considered in the form $S c=4 \pi m \zeta /\left(\rho D^{2} L\right)$. It is worth noting that even if the $Q S$-theory refers to the $n$-form of the mass ratio expression, $\rho D^{2} L / 2 m$, mostly employed in air flow, the previously defined form will be used, being the conversion formula in the case of rectangular bodies $m^{*}=D /(B 2 n)$. In aeroelastic oscillations the contribution of damping and $Q S$ forcing terms are comparable. However, it is important to remember that, given the water on air density ratio $\rho_{w} / \rho_{a} \approx 800$, the only negligible term is damping in water, resulting in a forcing term proportional to the increased mass ratio.

The lateral force coefficient is calculated here according to the form originally reported in Parkinson and Brooks (1961), that is

$$
C_{\mathrm{Fy}}(\alpha)=-\sec (\alpha)\left(C_{\mathrm{L}}(\alpha)+C_{\mathrm{D}}(\alpha) \tan (\alpha)\right)
$$

This can be determined by testing each stationary rectangular section's cylinder for several angles of attack in the vicinity of $\alpha=0^{\circ}$. QS theory states that galloping is driven by the instantaneous angle of attack between the body and the flow, $\alpha=\tan ^{-1}(\dot{y} / U$ ) (see Fig. 1), producing an asymmetric pressure distribution. The predictive mathematical model approximates the $C_{\mathrm{Fy}}$ vs. $\alpha$ with an odd polynomial in $\tan (\alpha)=(\dot{y} / U)$, whose first coefficient $A_{1}$ represents the slope of the lateral force coefficient $C_{\mathrm{Fy}}$ around zero angle of attack (Eq. (6)).

$$
A_{1}=\left.\frac{d C_{\mathrm{Fy}}}{d \alpha}\right|_{0^{\circ}}
$$

Despite its apparent simplicity, $A_{1}$ has shown a large variability in the reference literature, as recently shown in the survey reported by Mannini et al. (2014).

Cross-sections of concern in the present work have been demonstrated to be particularly prone to the interaction between VIV and galloping, although this has been for relatively low Sc values (at least well beyond the values characterizing experiments in water). This interaction was first observed by Parkinson and Brooks (1961) (Fig. 7 in their original paper) when a low damped square cylinder was found to start oscillating at the critical velocity of the Kármán-vortex resonance speed, instead of that predicted by the $Q S$ theory. Later, the interaction was observed and studied in rectangular cylinders with various SR values, e.g. in Smith (1962) and Novak and Tanaka (1974). It was also reported by Novak (1972) that rectangular sections with $S R>1$ are more prone to the interaction. It has been derived that the interaction occurs when the ratio $U_{g}^{*} / U_{r}^{*}$ is lower than a certain threshold, though the reporting of this shows some variability.

Parkinson and Wawzonek (1981) found a value of $\Lambda=U_{g}^{*} / U_{r}^{*}=2.15$, was necessary to separate the phenomena, even if some of the interaction effects were still present for $\Lambda \stackrel{g}{=}$ 8.4. A certain interference between VIV and galloping was found to be inseparable when $U_{g}^{*}<U_{r}^{*}$, a condition that was verified for light, slender low-damped structures in airflow and necessarily in water flow given the different flow density (Parkinson, 1989). In such conditions an effect referred to as quenching is exerted by the vortex structure on the body until it reaches $U_{r}^{*}$. This concept comes from non-linear dynamics (asynchronous quenching (Minorsky, 1947)) which was highlighted by Santosham (1966) and Bouclin (1977).

The interaction can manifest itself differently according to experimental conditions and dynamical system properties, e.g. in some cases the galloping excitation was seen to start at velocities corresponding to the Kármán-vortex resonance speed, instead of 5-6 times larger, or at a flow speeds larger than $U_{r}^{*}$ but less than one-third of that predicted by the theory of Santosham (1966) and Parkinson and Wawzonek (1981). Santosham (1966) conducted experimental tests to provide an insight into the reasons for such a behaviour. The failure of $Q S$ theory up to very high values of the reduced flow speed and large amplitudes of oscillation was suggested to be due to the effect of interacting Kármán vortices. Bouclin (1977) investigated a certain range of mass ratios for a square cylinder $(S R=1)$, using water flow measurements, and only one value of mass ratio for a $S R=2$ rectangular cylinder. They found the instability onset of the square cylinders quickly develops into galloping oscillations, and this occurred before $U_{r}^{*}$. This appeared to be a direct result of the interaction with vortex shedding 
and its onset depended on the mass ratio. A secondary resonance (super-harmonic) was found near $U_{r}^{*} / 3$, and was more evident for the rectangular section case.

The first wide parametric study of different geometries (sharp-edged rectangles, D-shaped sections and triangles) and damping ratios, was carried out by Bokaian and Geoola (1983) in water. The incoming flow turbulence intensity was also varied. The results showed a strong influence of turbulence intensity on the lateral force coefficient's form and characteristics, but not on the vortex-induced vibrations results nor their interaction with the galloping instability. However, the $S R$ and the damping factor strongly influence the response in amplitude.

Bokaian et al. (1984) also performed a detailed parametric study on the effect of varying the corner radius ratio for the square cylinder. In addition, they tested several values of the response parameter $K_{s}=\zeta /\left(n U_{r}^{* 2}\right)$, a different mass-damping parameter's form dependent on the aerodynamics of the section through the presence of $S t$ in the $U_{r}^{*}$ formulation. This was important given the variation of $S t$ with the corner radius ratio. It was observed that for low values of the response parameter $K_{s}$ the vortex resonance was inseparable from galloping, whereas a complete separation was observed for higher values of the $K_{s}$ parameter, leading respectively to a poor or a reasonable response in its amplitude prediction by using the QS galloping formulation. It was also noticed that, besides the aspect ratio $(A R=L / D)$ and $K_{S}$ as reasonably expected, the response strongly depends on the Reynolds number.

Another comprehensive study was conducted in Bokaian and Geoola (1985) on an SR $=0.5$ sharp-edged rectangle. The authors performed a parametric study on the turbulence intensity and structural damping ratio. One of the main results of interest from their study was that for the first time such a section was demonstrated to be a soft-type oscillator with respect to the VIV-galloping interference. Differently from those of higher $S R$, which can gallop spontaneously from rest and are therefore called "soft-oscillators", rectangular cylinders with shorter after-bodies, say $0.375<S R<0.683$ according to Parkinson (1963), are referred as "hard-oscillators" in smooth flow. This means that they need an external quantum of energy to reach a basin of attraction of the limit cycle (Novak, 1972; Nakamura and Tamonari, 1977).

More recently, Nemes et al. (2012) showed that the interaction was approached when a square section was tested in water flow for varying angles of attack. Several transitions between different kind of instabilities, such as galloping, VIV, intermittent response or more complicated forms of instabilities were found, showing unexpected and peculiar characteristics as the flow incidence was varied. A previously unreported branch of instability was discovered with very high amplitudes of oscillation. Subsequently, the analysis was focused on the three most interesting orientations of the square cylinder, namely $\alpha=0^{\circ}, 20^{\circ}$ and $45^{\circ}$ (Zhao et al., 2014a). These three angles correspond to the situations where the responses are dominated respectively by galloping, VIV and a more complex modified VIV. The third test case response appeared as a VIV response experiencing four response regimes: an initial branch (IB), an upper branch (UB), a higher branch (HB), and a desynchronization region, where the amplitude is approximately constant. This constitutes an interesting result with respect to what will be shown in the following.

For airflow measurements, there is interest in the $\alpha$-variation of a square prism by Hémon (2012), particularly concerning the possibility of a low-damped system in being able to lower the galloping instability onset velocity. This could find applications in energy harvesting purposes. An updated review and some new experimental tests on the interference between VIV and galloping in air were given in Mannini et al. (2014, 2016). These studies were focused on $S R=1.5$, and its peculiar features and strong susceptibility to interference was shown. Underlying the importance of this work are the implications in actual codes' prescriptions from the practical engineering point of view. The typical response consists in the principal instability branch taking place at $U_{r}^{*}$ for the entire range of mass-damping ratios investigated (note that the overall Scruton number range tested, collected from a number of different papers, was $4 \leqslant S c \leqslant 238$ ). A secondary selflimited excitation was observed at low reduced flow speed $\left(0.25 \leqslant U / U_{r} \leqslant 0.5\right)$, that disappeared for a certain $S c$ value (somewhere in the range $6 \leqslant S c \leqslant 15$ ). This secondary resonance is discussed in Mannini et al. (2016), and ascribed to the resonance with a secondary mode of vortex shedding (e.g. Shiraishi and Matsumoto, 1983).

The study proceeds by describing the experimental methods in Section 2. The results and discussion, including the Strouhal number and the transverse lift on a fixed cylinder, types of the structural response, and analyses of the oscillation amplitude and frequency, are presented in Section 3 and discussed in Section 4. Finally, conclusions and some outlooks for future studies are drawn in Section 5. The summary above highlights the complexity of the interaction between the flow-induced vibration modes and how this can significantly affects the body's response. This is clearly what motivated the study and determined the choices made for the independent variables.

\section{Experimental methods}

\section{Experimental apparatus}

The experiments were conducted in the free-surface recirculating water channel of the Fluids Laboratory for Aeronautical and Industrial Research (FLAIR) in the Department of Mechanical and Aerospace Engineering at Monash University. Fig. 2 shows pictures of the experimental set-up and the air-bearing system that was employed to model the hydroelastic system. The water channel facility has a test section of $4000 \mathrm{~mm}$ in length, $600 \mathrm{~mm}$ in width and $800 \mathrm{~mm}$ in depth. This water channel permits the user to conduct experiments in smooth flow, characterized by a longitudinal turbulence intensity lower than $1 \%$. Further details on this water channel facility and the air bearing system can be found respectively in Sherry et al. (2010) and Nemes et al. (2012) and Zhao et al. (2014a, b). The rigid rectangular cylinder model used was made from aluminium 
(a)

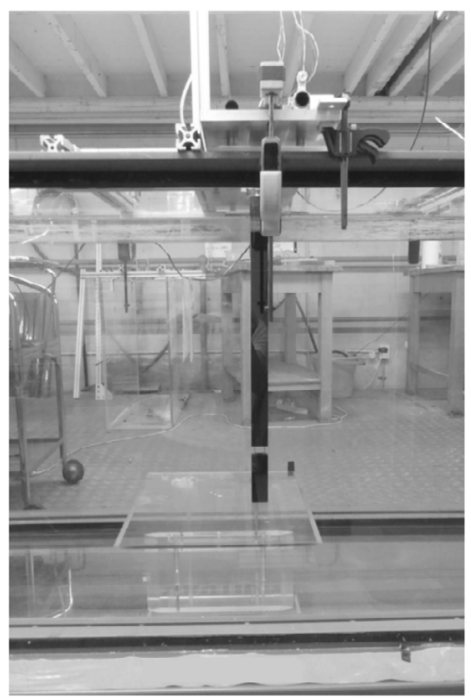

(b)

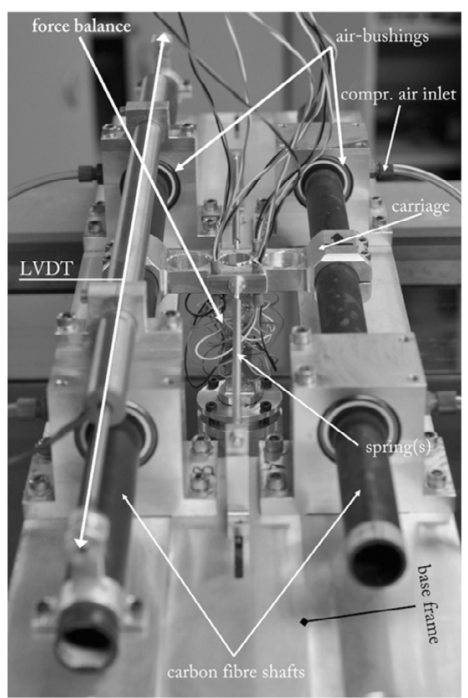

Fig. 2. The present experimental set-up: (a) side view of the rectangular cylinder model immersed in the water channel, and (b) components of the air-bearing system employed.
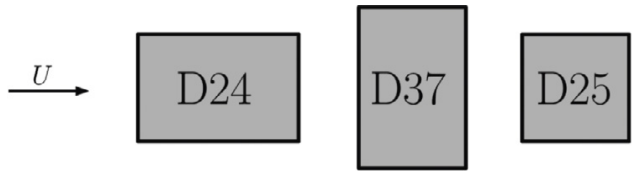

Fig. 3. Two main orientation of the rectangular section and labels used to refer to them: $\alpha=0^{\circ}$ corresponds to D24 and $\alpha=90^{\circ}$ corresponds to D37. D25 is the label used to refer to a square cross-sectional model.

Table 1

Specifications of the rectangular cylinder model used. The orientations the cylinder with $S R=1.5$ and 0.67 , are referred to as D24 and D37, respectively.

\begin{tabular}{llll}
\hline & D24 & D37 & Unit \\
\hline Side width $D$ & 24.75 & 37.45 & $\mathrm{~mm}$ \\
Side width $B$ & 37.45 & 24.75 & $\mathrm{~mm}$ \\
Side ratio $(S R=D / B)$ & 1.5 & 0.67 & - \\
Immersed length $(L)$ & 617.50 & 617.50 & $\mathrm{~mm}$ \\
Wall thickness $(t)$ & 2.14 & 2.14 & $\mathrm{~mm}$ \\
$A R=L / D$ & 24.90 & 16.47 & - \\
Blockage & 4.2 & 6.4 & $\%$ \\
Total oscillating mass $(m)$ & 1280 & 1280 & $\mathrm{~g}$ \\
Mass of displaced fluid $\left(m_{\mathrm{d}}\right)$ & 572 & 572 & $\mathrm{~g}$ \\
Mass ratio $\left(m^{*}\right)$ & 2.24 & 2.24 & - \\
\hline
\end{tabular}

tubing with cross-sectional dimensions of $D=24.75 \mathrm{~mm}$ and $B=37.45 \mathrm{~mm}$ (these rectangular sectional models will be from here on identified respectively by D24 and D37) and a wall thickness of $2.14 \mathrm{~mm}$.

The immersed length $(L)$ and aspect ratio $(A R)$ calculated based on side length, were $L=617.5 \mathrm{~mm}$ and $A R=25$. The total oscillating mass and the mass of displaced fluid were $m=1280 \mathrm{~g}$ and $m_{\mathrm{d}}=572 \mathrm{~g}$, respectively, giving a mass ratio of $m^{*}=m / m_{\mathrm{d}}=2.24$. The detailed specifications are shown in Table 1 . The starting position is with the shorter side perpendicular to the oncoming flow $\left(\alpha=0^{\circ}\right)$, nominating such a reference configuration as D24 corresponding to the side ratio of $S R=1.5$, whereas if it was rotated to $\alpha=90^{\circ}$ the cylinder has a side ratio of $S R=0.67$, and is identified as D37 (Fig. 3). Additionally, a square cross-sectional model (D25 in Fig. 3), as studied in Nemes et al. (2012), will be introduced for comparisons in the last part of the paper.

The cylinder model was vertically supported by a force balance sting that was coupled with a carriage mounted on the pair of carbon fibre shafts of the air bearing system (see Fig. 2b). To promote parallel vortex shedding, an end conditioning platform, was adopted in the present experiments. Although the top and bottom surface do not share the same end condition, this technique has been demonstrated to be effective for this type of experiments (Khalak and Williamson, 1996; Zhao et al., 2014a, b). This platform had a height of $165 \mathrm{~mm}$ and a top plate with dimensions of $600 \times 400 \times 5 \mathrm{~mm}$, giving a small gap of approximately $1 \mathrm{~mm}$ between the cylinder free end and the top plate. The cylinder oscillations were elastically mounted 


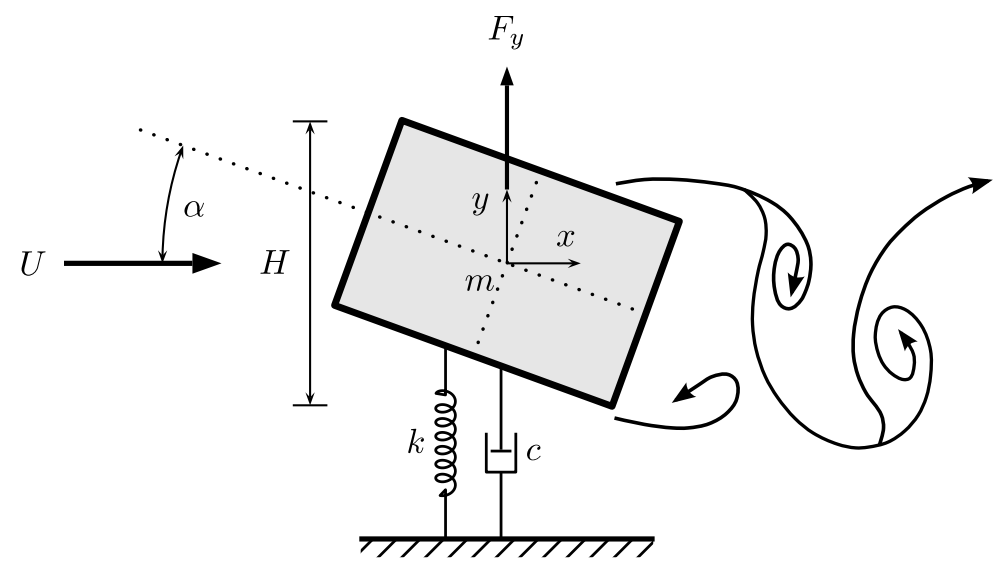

Fig. 4. Schematic of experimental rig showing the rectangular cylinder $(S R=1.5)$ with variable angle of attack, $\alpha$, and projected length, $H$, freely oscillating in the transverse direction, $y$, to the oncoming flow, $U$ in the stream-wise direction $x$. The body is mounted and elastically constrained to provide $k$, the system spring constant, and $c$, the structural damping.

by a pair of extension springs, and the shafts constrained the body to move with a single degree of freedom in the transverse direction to the oncoming flow. Typical values of mechanical damping (with respect to critical) identified through decay tests in still water, were around $0.26 \%$, while each spring is characterized by a stiffness of $0.01 \mathrm{~N} / \mathrm{mm}$. A linear variable differential transformer (LVDT) was used to measure the displacement of the cylinder with an accuracy of $1 \mu \mathrm{m}$ (see Nemes et al., 2012; Zhao et al., 2014a). The lift and drag forces acting on the cylinder were measured using a multi-component force balance having an accuracy of $5 \mathrm{mN}$ (see Zhao et al., 2014a, b). For the lift force calculation, the inertial force associated with the body motion was taken into account to yield the instantaneous fluid force, $F_{y}(t)$. To obtain a reference, the zero position of the cylinder was measured for each test in a quiescent fluid. Reference values of the forces were obtained in the same way.

\section{Results}

\subsection{Static tests}

The aerodynamic characterization of each section is obtained by differently orientating the cylinder through static tests. Results of force measurements are employed here to detect two nondimensional parameters, the Strouhal number (St) and the $A_{1}$ parameter, which has been already introduced and is given by $\left.\left(d C_{\mathrm{Fy}} / d \alpha\right)\right|_{0^{\circ}}$. To perform such tests the shafts supporting the model in the air bearing system were clamped to the channel walls so as to prevent any transversal motion. The force balance connecting the model to the rigid arm was fixed to the moving part of the system in a way that was rigid enough for the purposes of these tests. It was expedient to set the desired angle of attack early in the experimental campaign by manually imposing it and ranging from $-5^{\circ}<\alpha<+95^{\circ}$, without imposing a fixed step angle and refining the measurements around $\alpha_{0}$. Given such a large $\alpha$-range, a modification in the rig was aimed at a more refined $\alpha$-discretisation and a shorter amount of measurement time. The experimental rig used was originally designed to investigate the transverse FIV of a circular cylinders under forced rotation by Wong et al. (2017); Zhao et al. (2018b) (Fig. 5) and here is referred to as the rotational rig. The rotational rig consisted of a rotary motor that could be screwed to the bottom of the force balance, in the same manner as the sectional model. After inserting the model inside it by means of an aluminium adapter, it was fastened with four lateral grub screws. The aluminium adapter was designed and manufactured with great care to prevent wobbling or other undesired effects.

The rotor was externally controlled using a Parker 6K2 motion controller, which allowed us to impose the desired angular position or constant velocity on the cylinder. Here it was programmed to obtain angular rotations instead of velocities. The procedure for the detection of effective angles was made independently of the precise assignment of the perfect $\alpha=0^{\circ}$ position. The cylinder was simply adjusted in a reference near-zero position, then it was programmed to automatically start from a negative angle $\left(\alpha=-5^{\circ}\right)$ and moving with a fixed angular step of roughly $\Delta \alpha=0.5^{\circ}$ up to $\alpha=100^{\circ}$, that was $\alpha=-10^{\circ}$ for the $S R=0.67$ case. In this way the angle of attack range was well discretized.

Each position assumed by the cylinder was captured using a PCO2000 camera (2072 pixel $\times 2072$ pixel) placed exactly under the cylinder and beneath the water channel floor. It was triggered with the motion controller by sending a TTL signal for each change in the angular position. Once the TTL signal was received an image was automatically saved through the camera control software. This approached saved considerable time.

Once the resulting images and signals were obtained the real values of each angle was determined by digitally postprocessing the images using customized Python codes, comparing each actual image with the initial one, originally captured at the nominal zero angle. 


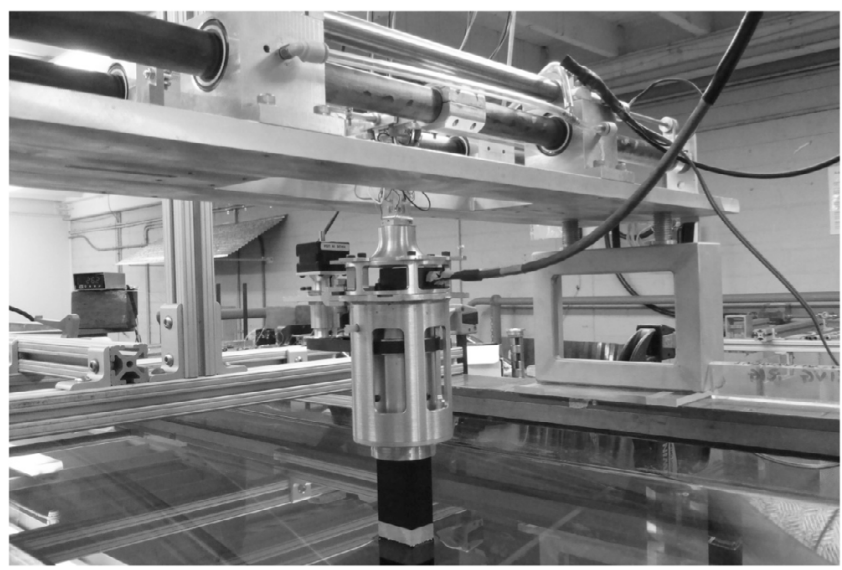

Fig. 5. Rotor rig view from the water surface level.

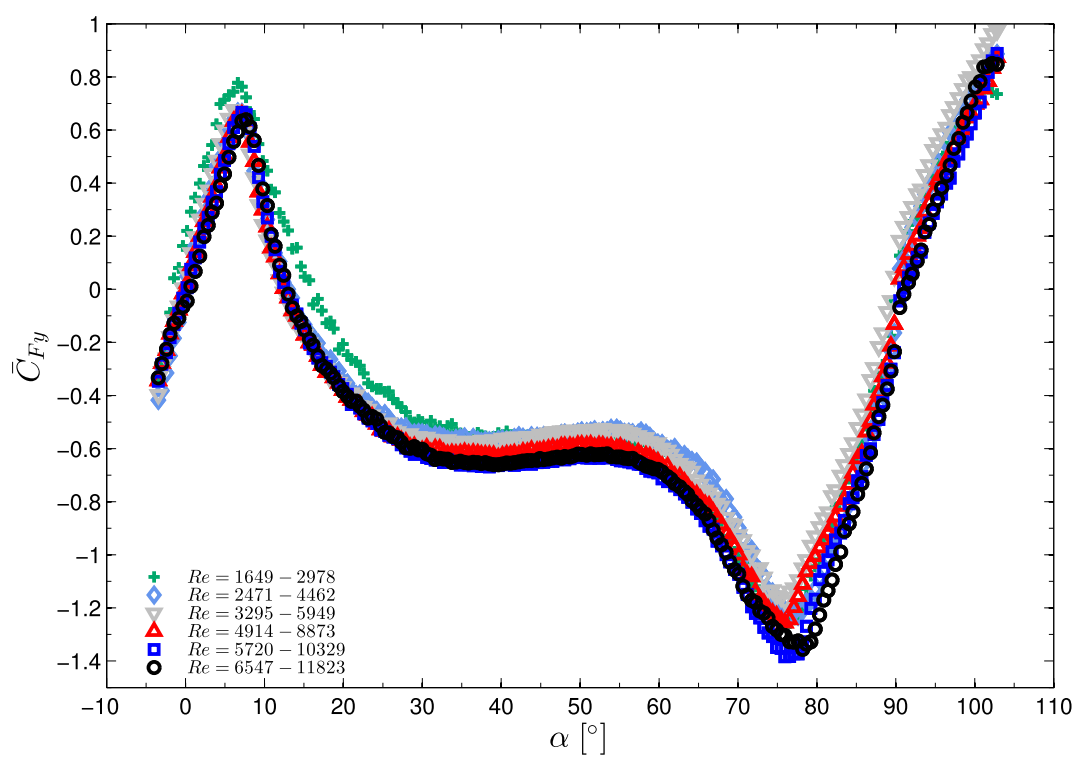

Fig. 6. Measurements of $\bar{C}_{\mathrm{Fy}}$ for the entire range of $\alpha$ tested though the rotor rig.

The entire $\alpha$-range $\left(-5^{\circ} \leqslant \alpha \leqslant 110^{\circ}\right)$ investigated is shown in Fig. 6 for six free-stream velocities (corresponding in turn to six Re-ranges since Reynolds number is defined on the projected area, see later), so that a subtle Re-dependency could be better observed in Fig. 7a for the $S R=1.5$ case. The only exception is at the lowest flow velocity, showing apparent deviations of the $\bar{C}_{\mathrm{Fy}}$ curve from the cases at higher velocities. This is probably because the flow exerts very low forces on the gauges for this case, which may be near the sensitivity of the measurement system. The Reynolds number used here is defined by $R e=U H / v$, where $H$ is the frontal projected height $(H=D \cos (\alpha)+B \sin (\alpha)$, see Fig. 4) and $v$ is the kinematic viscosity of the fluid. The $S R=0.67$ case in Fig. $7 \mathrm{~b}$ appears to be more $R e$-sensitive. This may be simply due to a stronger response to the vortex shedding by the wider side facing the flow, in addition to the slightly lower stiffness offered by the rotational rig, as the presence of the rotor backlash does not guarantee a perfect fixed-end constraint.

Using the above-mentioned procedure, static force measurements were used to calculate $A_{1}$, which is a measure of the proclivity of a section to galloping instabilities. Both the $S R$ cases were found to be a soft-type oscillator in water flow, in agreement with the above-mentioned results reported by Bokaian and Geoola (1983). This is apparent from Fig. 7, where both the cases show a positive slope of $C_{\mathrm{Fy}}$ around the zero angle of attack, making the body prone to the instability from rest. Not reported here are the results for a square section, as it has already been demonstrated to exhibit the same behaviour in the first paper by Parkinson and Smith (1964). For the rectangle with $S R=1.5$, labelled here D24, the order of magnitude of the maximum reached by $C_{\mathrm{Fy}}$ is consistent with previous results (see Fig. 5 in Mannini et al. (2016) for experiments in air). 

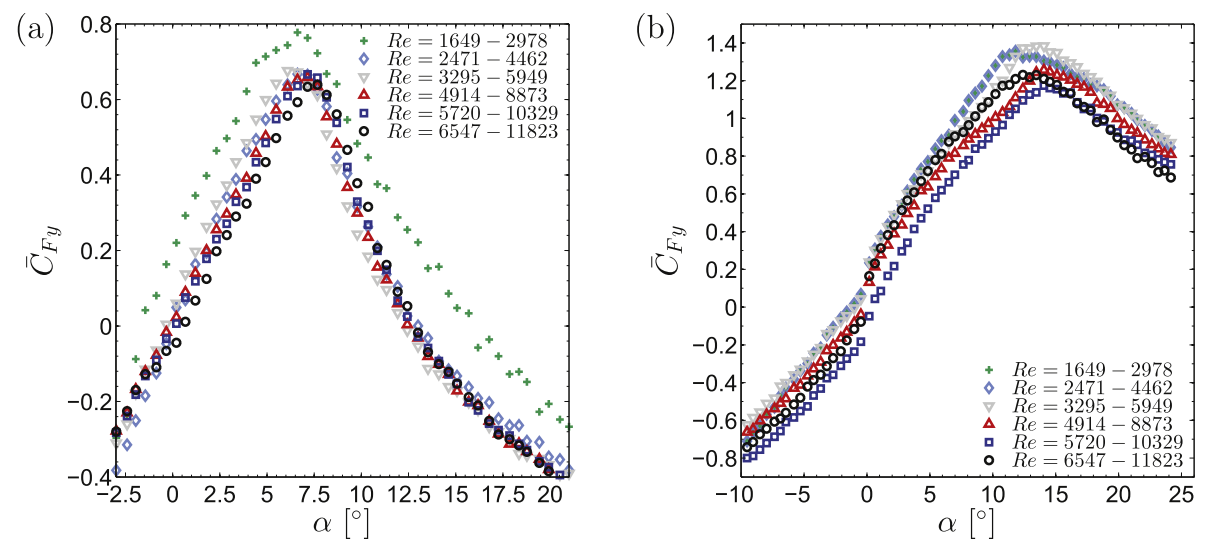

Fig. 7. Reynolds number dependency of the lateral force coefficient $\bar{C}_{\mathrm{Fy}}$ for the D24 and D37 cases in (a) and (b), respectively. The (·) notation indicates the mean values considered in calculating the lateral force coefficient $C_{\mathrm{Fy}}$.

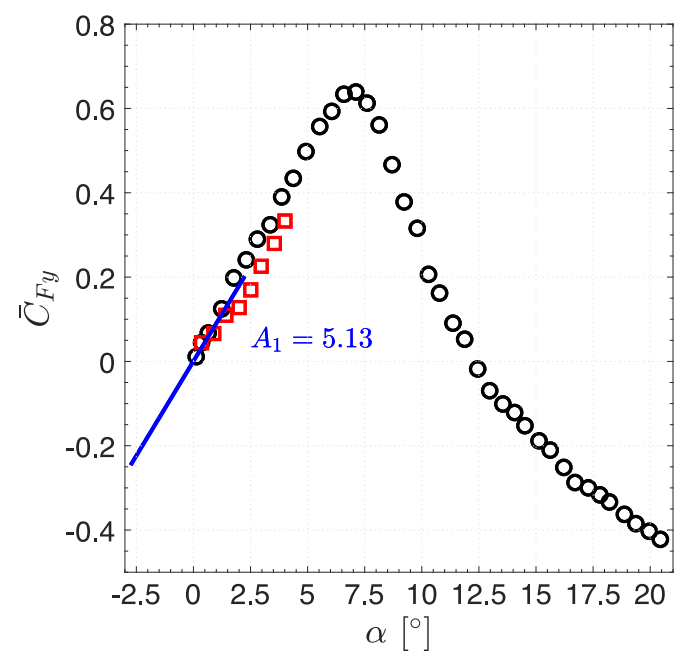

Fig. 8. Evaluation of $A_{1}$ parameter from the slope (in blue line) of lateral force coefficient $C_{\mathrm{Fy}}$ for the maximum $R e$ of the $S R=1.5$ (D24) case. The red squares represent negative angles of attack mirrored in order to assess the perfect symmetry of the sectional model.

Nevertheless, it is apparent that there are differences compared to the usual curve profiles of $C_{\mathrm{Fy}}$ versus $\alpha$ reported in the literature. In both cases a linear trend continues until reaching the peak value corresponding to the apparent angle of attack from which the section experiences the reattachment of the shear layer on the side making the section stable. This value for $S R=1.5$ occurs before $\alpha \approx 7.5^{\circ}$ while for $S R=0.67$ at $\alpha \approx 13^{\circ}$. Parkinson (1963) gave a value of $\alpha \approx 7^{\circ}$ for a $S R=2$ rectangular section in air flow, while for a square section it was approximately $13^{\circ}$ (Parkinson and Smith, 1964). For $S R=1.5$ in airflow results reported in figures 3.17-3.18 of Massai (2015) and figures 20-21 in Mannini et al. (2016) for two different aspect-ratio models (different Re and cross-sectional dimensions) are at $\alpha \approx 9^{\circ}$.

By extrapolating the most reliable result for the case of $S R=1.5$, usually corresponding to the maximum $R e$ (higher loads are better measured by instruments), it is possible to find a value for $A_{1}$. The linear regression is reported in Fig. 8, together with the negative angle of attack values mirrored in the positive quarter, giving a measure of the model symmetry; apparently the $\alpha$-peak resulted occurred at $\alpha \cong 7.11^{\circ}$. The value of $A_{1}=5.13$ is finally proposed here for $S R=1.5$, whereas a value of $A_{1}=5.5$ has recently been reported in Mannini et al. (2016). An apparent feature shown in Fig. 8 is the lack of the inflection point reported in previous studies (e.g. Parkinson, 1963) for $S R=0.5$ and 1 cylinders; this has been seen by Massai (2015) and Mannini et al. (2016) for $S R=1.5$ in two different wind tunnel facilities. The soft-oscillator character of both sections has been mentioned above, and is reported in previous studies where measurements were made in water flow. Nevertheless, it is interesting to note that Novak and Tanaka (1974) presented data collected in smooth air flow measurements on a sharp-edged rectangle with the same side ratios (1.5 and 0.67), in which it is apparent that $S R=0.67$ resulted in a hard-type oscillator. However, for $S R=1.5$ the shape of the $C_{\mathrm{Fy}}$ versus $\alpha$ curve shows similar features to those reported in the present work. No inflection points are clearly visible, resembling the shape reported in Fig. 7a, although the maximum point is reached for $\tan (\alpha)=0.18 \Leftrightarrow \alpha \approx 10^{\circ}$. 

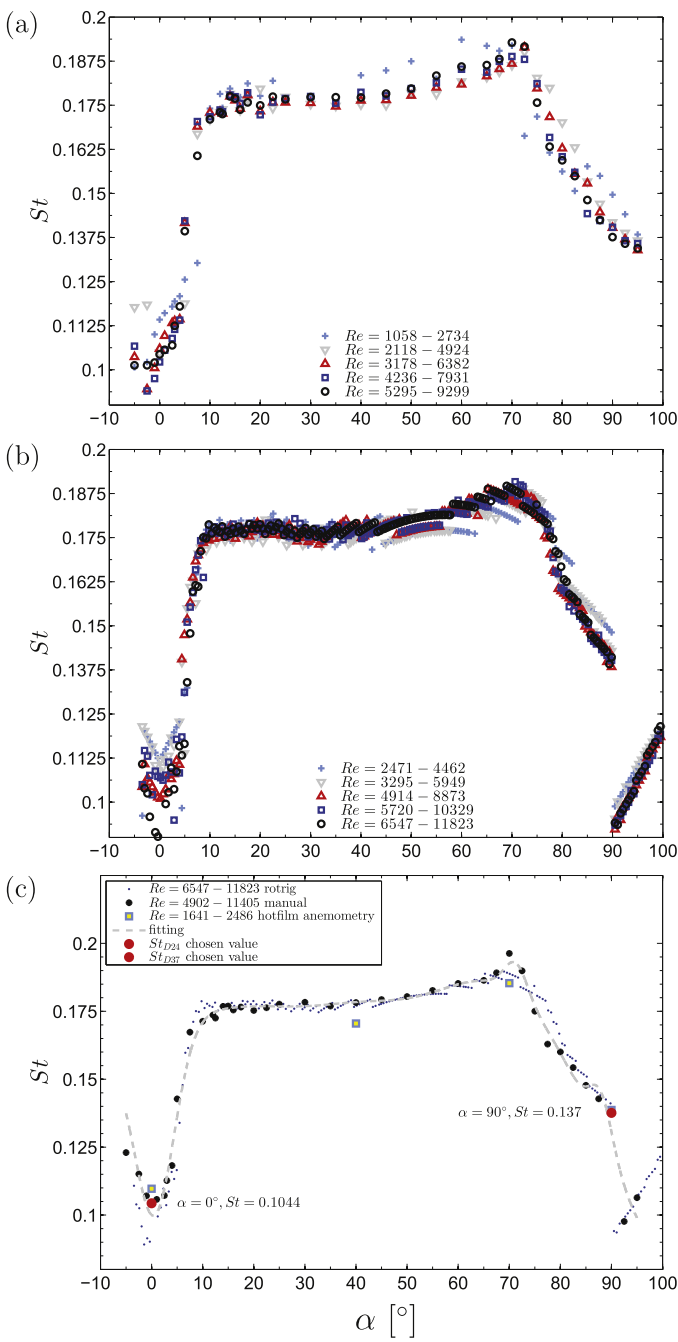

Fig. 9. Re-and $\alpha$-dependency of Strouhal number values determined by changing the angle manually (a) and automatically using the rotator rig (b). In the figure (c) maximum Re data of the two above showed techniques are reported together with hot-film anemometry results for four angles only, and with the chosen values at the two main orientations of the rectangular cylinder.

Particular attention was paid to the characterization of the Strouhal number, considering that it is directly linked to the definition of the critical velocity $\left(U_{r}^{*}=1 / S t\right)$. The spectral analysis of the transverse lift provides the $S t$ detection for each angle of attack, by picking up the lift spectral peak values for each test point. The Re-dependent results of $S t$ are shown in Fig. 9a and $b$ for the angle of attack setting modes of manual and automatic (via the rotational rig), respectively. There seems to be qualitative and quantitative agreement in the results for the two techniques; the major differences are (i) a lower $R e$-dependency for the first case (manual) and (ii) a more refined and wider $\alpha$-range investigated in the second (automatic). A hot-film anemometer was also placed $150 \mathrm{~mm}$ downstream with respect to the trailing edge of the $S R=1.5$ case $(\approx 1.2 D$ in the transverse direction in the near wake of the cylinder), at four angles of attack $\left(\alpha=0^{\circ}, 40^{\circ}, 70^{\circ}\right.$ and $\left.90^{\circ}\right)$. The most representative results are presented by coloured square markers in Fig. 9c, together with those referred to the maximum Re of the first two measurement techniques. Measurements using hot-film anemometry were also carried out for two flow velocities to confirm the results by recording at the same point for two different durations ( 5 and $20 \mathrm{~min}$ ). Good agreement between the different techniques was found, with $S t=0.104$ for $S R=1.5$ and $S t=0.137$ for $S R=0.67$. A value of $S t=0.131$ has been given in Zhao et al. (2014a) for a square section (D25) tested in the same water channel facility. The present results are in agreement with previous studies, e.g. $S t=0.108$ by Smith (1962) and $S t=0.106$ by Mannini et al. (2016) for rectangular cylinders with $S R=1.5$ 
(a)

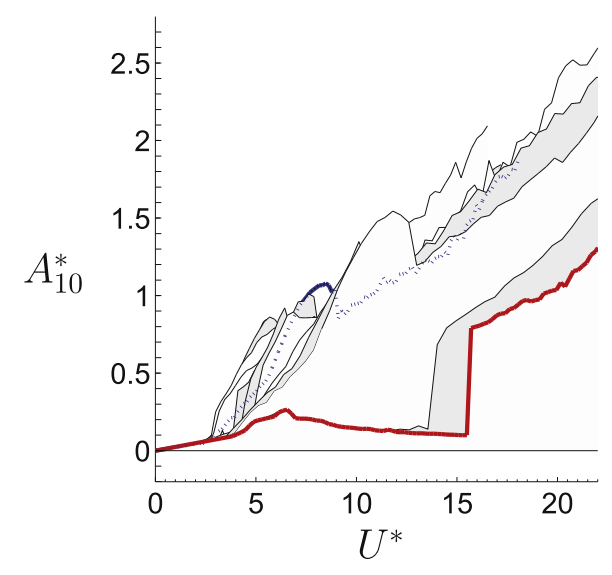

(c)

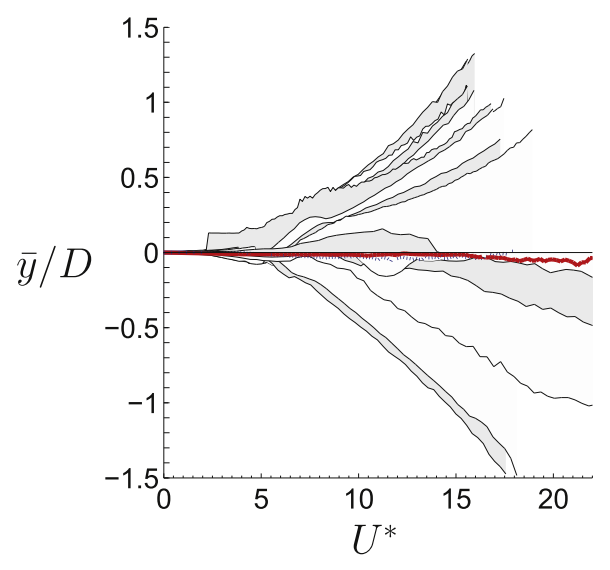

(b)

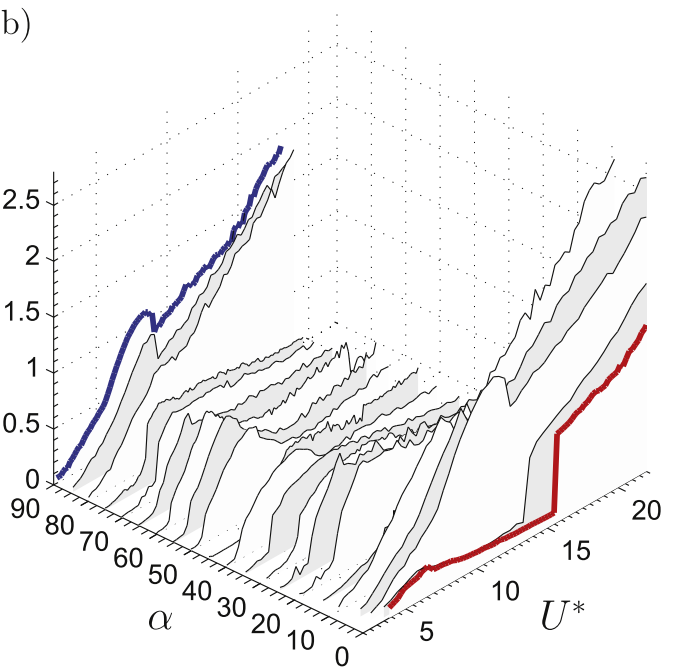

(d)

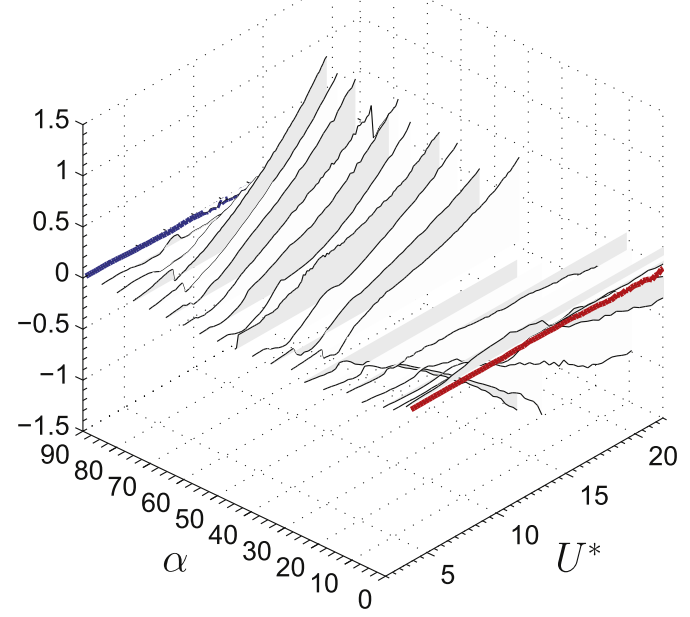

Fig. 10. The amplitude response $\left(A_{10}^{*}\right)$ as a function of reduced velocity, $U^{*}$, with all angles of attack, $\alpha$, superimposed in (a). 3D plot of amplitude response against $U^{*}$ and $\alpha$ in (b). The bold lines refer to the $S R=1.5$ case (blue bold line in the online version) and the $S R=0.67$ case (red bold line in the online version). (c) shows the mean amplitude response $(\bar{y} / D)$ against $U^{*}$ for varying $\alpha$ between $0^{\circ}$ and $90^{\circ}$, at $5^{\circ}$ increments, and the $3 \mathrm{D}$ view is shown in (d).

\subsection{Hydroelastic response}

Results from the hydroelastic campaign conducted with the $S R=1.5$ rectangular cylinder at a very low value of the mass ratio $m^{*}, 2.24$ (see Table 1), are reported in this section. The main response parameter considered as statistically representative of the signals recorded is the so-called $A_{10}^{*}$, defined as the mean of the top $10 \%$ of the peaks, and already used in Hover et al. (1998), Morse et al. (2008), Nemes et al. (2012) and Zhao et al. (2014a). To confirm its use the $A_{10}^{*}$ was compared with the maximal amplitude peak, referred as $A_{\max }^{*}$, to give additional information about how well they statistically compare in extracting results from pseudo-sinusoidal signals.

Types of structural response. Given the amount of data collected and the number of angles of attack tested, a useful and comprehensive results summary is presented using two- and three-dimensional plots of the $A_{10}^{*}$ and $\bar{y} / D$ response curves for the $\alpha$-range investigated (Fig. 10). This provides a global view on how varying the angle of attack changes the FIV response curves shape. In particular, plots in the left column of Fig. 10 are the 2D side views of the corresponding 3D plots (right column) seen from the $U^{*}-A_{10}^{*}$ plane. Higher $U^{*}$ values are neglected in this representation to have a close-up view on the global results, while the entire velocity range will be shown in the following. A net mean lift in asymmetric configurations is generated by the increase of the angle of attack. The $A_{10}^{*}$ response for increasing velocities and angles of attack (Fig. 10a and b) reveals different response shapes, here grouped into nominated Types, each one having common and identifiable features. Type I, is typical of $\alpha=0^{\circ}(S R=1.5)$, and $\alpha=1^{\circ}$, and is highlighted with a bold grey line for $\alpha=0^{\circ}$ (red in the online version). Type II response is typical of the $S R=0.67$ section, that is $\alpha=90^{\circ}$ (darker bold line and blue in the online version), 

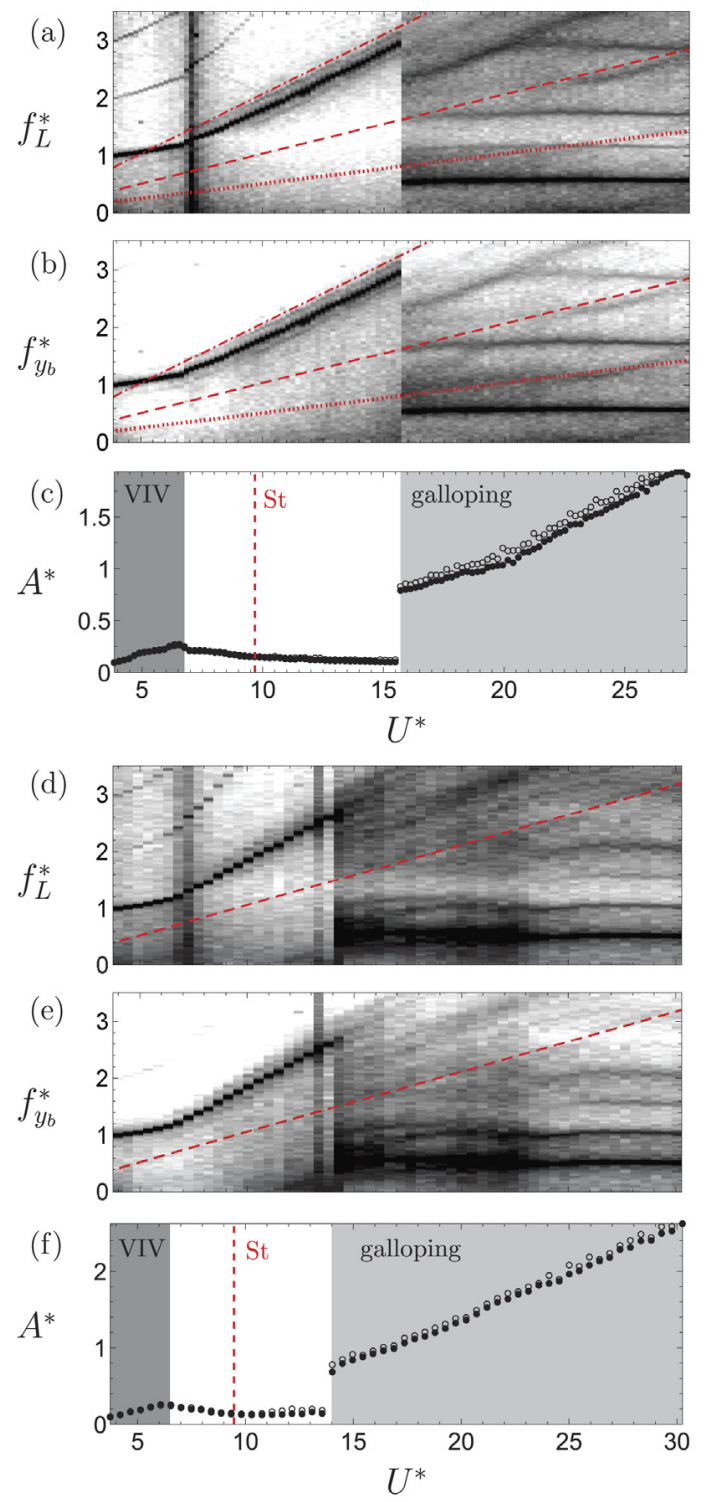

Fig. 11. Logarithmic-scale reduced frequency power spectrum contours of the lift coefficient $f_{L}^{*}$ (a) and of the body displacement $f_{y_{b}}^{*}(\mathrm{~b})$. In figure (c) the normalized amplitude response $A^{*}$ as a function of $U^{*}$ is reported. (a)-(c) figures are relative to $\alpha=0^{\circ}$ while (d)-(f) are relative to $\alpha=1^{\circ}$ configuration. Empty and filled markers respectively indicate $A_{\max }^{*}$ and $A_{10}^{*}$ response. Dark and light grey areas are labelled as VIV and galloping response branches respectively.

but also comprehensively covers $\alpha=2.5^{\circ}, 5^{\circ}, 10^{\circ}, 80^{\circ}$ and $\alpha=85^{\circ}$. The third, and largest range, Type III (angles ranging within $15^{\circ} \leqslant \alpha \leqslant 75^{\circ}$ ), exhibits different and mixed response features. The mean amplitude response $(\bar{y} / D)$ is also reported (Fig. 10c and d), providing information about the lifting direction of the different orientations.

Representative cases of each response Type will be investigated in detail to better show their similarities and differences, and, thence, try to understand the reasons behind the transitions clearly visible in Fig. 10b.

Type I response. Two cases at $\alpha=0^{\circ}$ and $1^{\circ}$, have been assigned to this category, and given their similarities it is of interest to analyse and compare them. In Fig. 11 the nondimensional amplitude response curves, in terms of $A_{\max }^{*}$ and $A_{10}^{*}$ are shown below two other plots expressing the logarithmic-scale reduced frequency power spectrum contours of two quantities, the lift coefficient $\left(f_{L}^{*}=f_{L} / f_{n}\right)$ and the body-displacement $\left(f_{y_{b}}^{*}=f_{y b} / f_{n}=f / f_{n}\right)$. These latter plots give us detailed characteristics of the instability that can be observed using the response curves graphs (namely Fig. 11c and f respectively for $\alpha=0^{\circ}$ and $1^{\circ}$ ): the frequency domain information helped in the definition of two areas, which are coloured in dark and light grey. The first (darker) region refers to a velocity range characterized by VIV response, while the second denotes a pure galloping response. 
In the frequency response contour plots a darker colour means a more pronounced peak in the spectrum of the signal, i.e. that there is higher energy contained in the response. Reference vertical straight lines (red-coloured in the online version) on the $A^{*}$ plots presents a vertical dashed line to show the reduced resonance velocity $\left(1 / S t_{0^{\circ}} \simeq 9.58\right)$. From static measurements the corresponding Strouhal numbers, found through spectral analysis of the lift force, are $S t_{0^{\circ}}=0.104$ and $S t_{1 \circ}=0.106$, these are shown in the frequency response contour plots with the same line-type. Only for $S R=1.5$ two additional lines in the $f^{*}-U^{*}$ plots, one dash-dot and one dotted lines, have been added indicating respectively $2 S t\left(f_{\text {vs }}\right.$ superharmonic) and $S t / 2\left(f_{\mathrm{vs}}\right.$ sub-harmonic) slopes. These are useful in comparing the slopes of the dominant frequencies shown by the darker traces.

For the VIV response region, the slope in nondimensional frequency response barely violates the Strouhal law, as is typical of the lock-in range, which is well known to be flattened in air flow because of the negligible added mass contribution (Sumer and Fredsøe, 1997). It is apparent that the lock-in region is well below the resonance velocity, and this constitutes the first peculiarity in the $S R=1.5$ case for such a low mass ratio. The dark vertical shadowing in $f_{L}^{*}$ plot (Fig. 11a) detects the peak in the amplitude response, followed by a desynchronization region $\left(7.5<U^{*}<15.5\right.$ for the $S R=1.5$ case) in which the maximum frequency response curiously follows the $2 S t$ slope, meaning a continually lowering oscillation amplitude with an increasing vibration frequency. This desynchronization region extends until there is a jump upwards in amplitude at $U^{*}=15.7$, where there is a corresponding sharp drop in frequency response. After this the reduced frequency appears to be stable at a value of 0.5 and remains stable over the rest of the $U^{*}$ range investigated. This $f^{*}$ value is the half that usually obtained in air flow $\left(f \simeq f_{n}\right)$.

Within the galloping response region the signals are more noisy than in the synchronization and desynchronization ranges, as evidenced by the diffused dark grey around the dominant frequency line. Also, there is the more marked presence of higher harmonics which interact with each other. In the galloping region in Fig. 11a, the higher 2nd, 3rd and 6th harmonics of $f^{*}$ are clearly visible. The 6th harmonic interacts with a $f_{\text {vs }}$ super-harmonics, forming what appears to be a bifurcation trace in the frequency domain, which correspond to the $1.5 S t$ in slope. At this corresponding point, $U^{*}=20.6$, there appears to be an increase in the slope of $A^{*}$ response. From a closer examination of the time histories of displacements (not reported here for sake of brevity) with the signals showing modulations with higher wavelength compared to those at lower velocities. A last remark about this fact is that the (practically linear) amplitude response in the velocity ranges $3.9 \leqslant U^{*} \leqslant 6.6$ (resonance region) and $15.7 \leqslant U^{*} \leqslant 20.6$ (first part of galloping region) appear to align on the same linear trend. In the $\alpha=1^{\circ}$ relative plots, for which less velocity points were recorded during experiments, it is apparent that the behaviour is similar, except for the evident lowering of the velocity threshold at which the above-described jump upwards in amplitude take place (from $U^{*}=15.7$ to $U^{*} \simeq 14$ ).

Such sensitivity in the response with respect to such a slight change in angle of attack warranted further investigation. A second set of tests was carried out at $\alpha=0^{\circ}$ after having dismounted and then remounted the same set-up and model to double-check repeatability, first increasing and then decreasing the flow velocity, to assess for any presence of hysteresis cycles. The results are reported in Fig. 12. In the central part of the figure the two amplitude $\left(A_{10}^{*}\right)$ curves with increasing and decreasing flow velocity are shown. In the figure, the larger markers identify the points for which time histories were taken. They are labelled in alphabetical order and referred to increasing velocities. It is apparent that the jump upwards is localized and occurs in the proximity of $U^{*} \simeq 20$, which is different from the same test case curve proposed in Fig. 11c. From the time traces (Fig. 12(a)-(f)) there appears to be a "competition zone" ranging from $U^{*} \simeq 15.7$ to $U^{*} \simeq 20$ in which it is likely that two branches, somewhat unstable, are competing with each other, resulting in the solution switching to one state to another. The recorded signals here are $5 \mathrm{~min}$ long, and the waiting time before increasing/decreasing velocity was around $2 \mathrm{~min}$. (The statistical convergence of the signals were also double-checked with longer measurements.) The damping ratio for this test case was slightly higher (0.3\% against $0.26 \%$ ) than in the previous case, and may be a reason for this delayed switch from desynchronization to the galloping response branch. For these reasons it seems unlikely to be profitable to discuss at length any hysteresis behaviour in this case. Such behaviour was not observed for $\alpha=90^{\circ}$, in fact the curves recorded with increasing and then decreasing flow velocity overlaps entirely.

Type II response. When the angle of attack reaches $\alpha=2.5^{\circ}$, even though the hydrodynamics do not appear to change noticeably $\left(S t_{2.5^{\circ}}=0.108\right)$, the hydroelastic response completely changes and exhibits different shapes and features. Recalling the 3D response graph (Fig. 10) the most peculiar feature is apparent: the amplitude response curve shape resembles that of $S R=0.67$, which corresponds to $\alpha=90^{\circ}$ with $S t_{90^{\circ}}=0.137$. From an examination of the whole $\alpha$-range, the test cases included in the Type II response are the angles of attack in the upper $\left(80^{\circ} \leqslant \alpha \leqslant 90^{\circ}\right)$ and lower $\left(2.5^{\circ} \leqslant \alpha \leqslant 10^{\circ}\right)$ ranges. It is interesting to compare (as proposed above Fig. 11), the most representative case $\alpha=90^{\circ}$ and the case $\alpha=2.5^{\circ}$ (Fig. 13). These two cases appear to have almost identical response curves for both the amplitude and frequency responses. Clearly, this response type is completely different from Type I: the instability developed from the lowest velocities in the synchronization region, where the oscillation frequency tends to closely follow the Strouhal vortex shedding frequency, prior to the lock-in region (marked in dark grey). In the galloping-dominated region at higher $U^{*}$ values, the amplitude response tends to increase linearly, while the oscillation frequency is split into two components, with one close to $f_{\mathrm{n}}$ and the other following the trend of the Strouhal vortex shedding. This region develops directly from the instability branch. Remarkably, in contrast to near-zero angles of attack, harmonic frequencies are clearly visible in the synchronization region, while the galloping region is characterized by nonperiodic oscillations evidenced by broadband noise in the frequency response. 
(a)

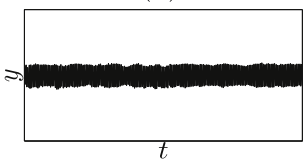

(d) (b)

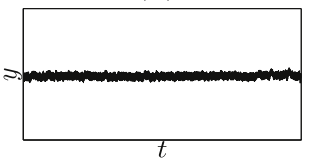

(e) (c)

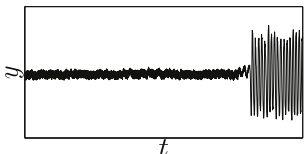

(f)
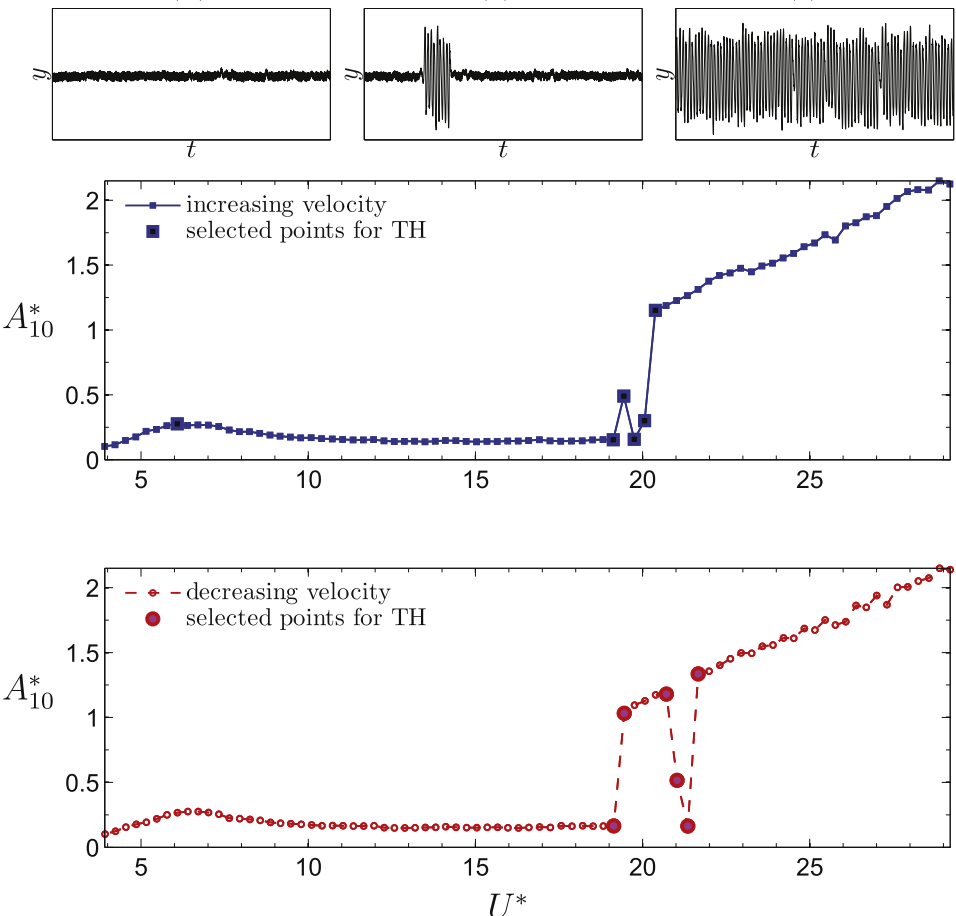

(g)

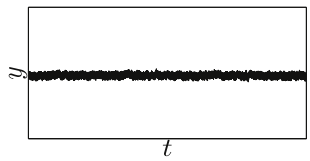

(h)

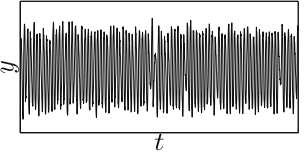

(i)

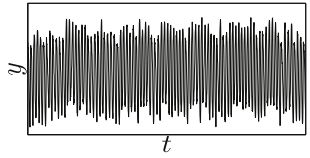

(j)

(k)
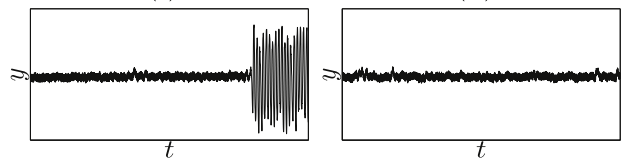

(l)

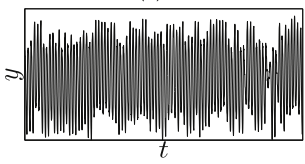

Fig. 12. Selection of time traces reporting the intermittent behaviour in terms of oscillation amplitude in proximity of $U^{*}=20 \simeq 2 U_{r}^{*}$ : (a)-(f) and ( $)-(\mathrm{l})$ series have been both ordered for increasing velocity points.

Type III response. In the range $15^{\circ} \leqslant \alpha \leqslant 75^{\circ}$, there are a number of different mixed response types. More precisely this response type should be divided into, at least, two subgroups as will be shown below. The reason for including all these orientation cases in one group is that they never gallop, or, at least, no clear galloping trends were observable within the tested $U^{*}$ range. Hence, the FIV response appears to be somewhat chaotic in this $\alpha$ range.

Fig. 14 shows the amplitude and frequency responses as a function of $U^{*}$ for three different cases of $\alpha=20^{\circ}, 45^{\circ}$ and $50^{\circ}$ (the same as previously presented), with each of them showing different, particular features. The cases $\alpha=20^{\circ}$ (Fig. 14a-b) and $\alpha=50^{\circ}$ (Fig. 14e-f) present significantly different amplitude responses. In the $\alpha=20^{\circ}$ case, a clearly VIV region is observed for $5.6 \lesssim U^{*} \lesssim 7.4$, where the frequency response, deviating from the Strouhal vortex shedding frequency trend, comprises multiple harmonics. Beyond this the dominant frequency tends to the trend of the Strouhal vortex shedding frequency (the red dashed line) at higher $U^{*}$ values, which is indicative of desynchronization. The main differences with respect to the case of $\alpha=50^{\circ}$ are the onset and the $U^{*}$ range of the resonance, the presence of a larger number of $f_{\mathrm{vs}}$ harmonics, and the $f^{*}$ response features of the desynchronization region. In fact, for $\alpha=50^{\circ}$ the lock-in region 

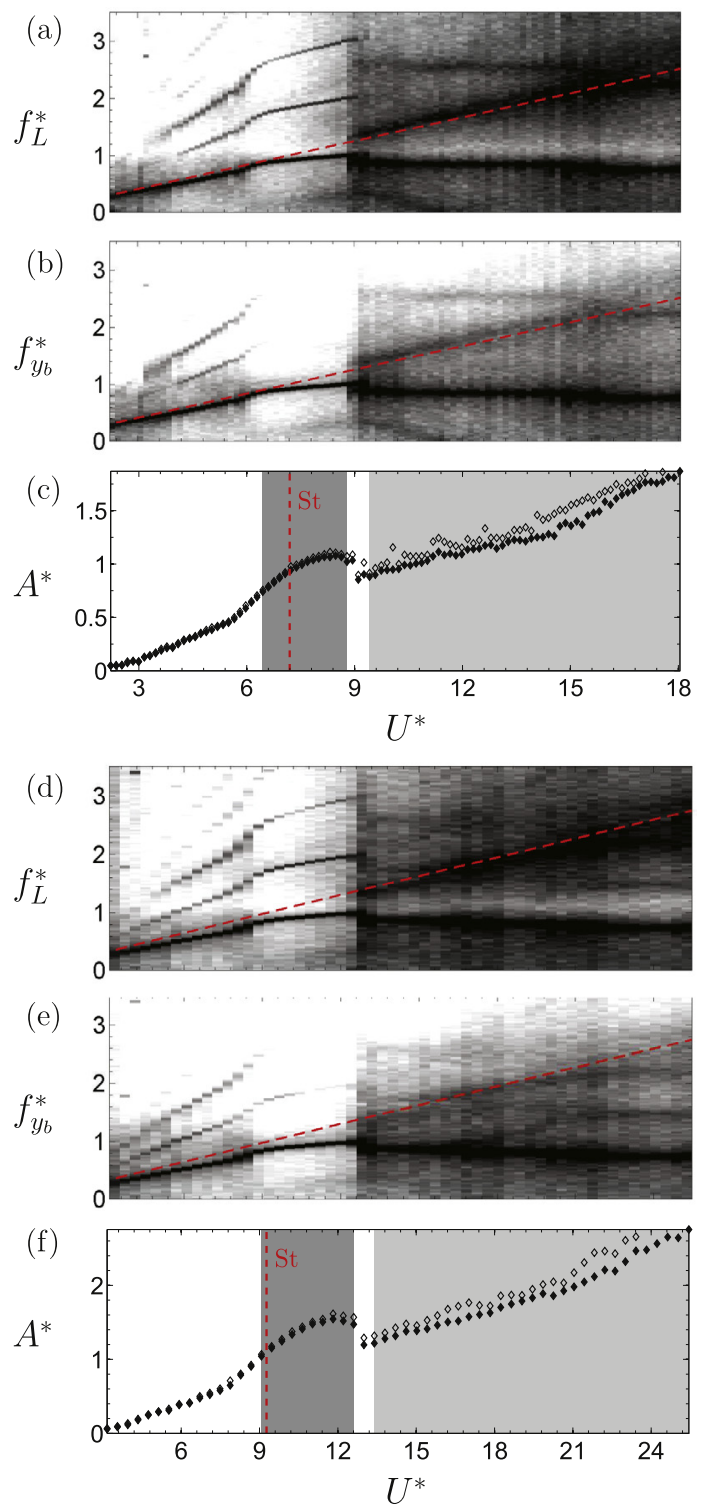

Fig. 13. (a) Logarithmic-scale reduced frequency power spectrum contours of the lift coefficient $f_{L}^{*}$ and (b) body-displacement responses $f_{y_{b}}^{*}$. (c) The normalized amplitude response $A^{*}$ for the $S R=0.67$ cylinder at $\alpha=90^{\circ}$ as a function of $U^{*}$; (d), (e) and (f) are relative to $\alpha=2.5^{\circ}$. Open and filled markers represent the $A_{\mathrm{max}}^{*}$ and $A_{10}^{*}$ values, respectively. Dark and light grey zones stay for VIV and galloping range respectively as in Fig. 11 .

occurs at a lower $U_{r}^{*}$, in accordance with the $f^{*}$ response, and also the desynchronization region begins with a decreasing amplitude response.

Furthermore, in addition to the 2nd and 3rd harmonics of $f_{v s}$, which are also evident in the case $\alpha=20^{\circ}$, the sub-harmonic $f_{\mathrm{vs}} / 2$ and harmonics of $3 f_{\mathrm{vs}} / 2$ and $5 f_{\mathrm{vs}} / 2$ are present within the synchronization region at $\alpha=50^{\circ}$. Because of this these two cases are referred to as Type IIIa.

For $\alpha=45^{\circ}$ shown in Fig. 13c-d, the dominant oscillation frequency component appears to be close to the Strouhal vortex shedding frequency, with other harmonics present clearly in the range of $7 \leqslant U^{*} \leqslant 9.5$. In this region, the body oscillation frequency is synchronized with that of the vortex shedding. Also, there is a jump in the amplitude response associated with the onset of the synchronization region. This case has been categorized as Type IIIb response. The cases of $\alpha=15^{\circ}$ and $75^{\circ}$ were found to be similar and fitted in this category of response type.

A global view on the above-described response Types collection of amplitude response curves is proposed in Fig. 15. The response parameter chosen here is the equivalent nondimensional sine wave amplitude signals $\left(\sqrt{2} y^{\prime} / D\right.$, where $y^{\prime}$ indicates the $r m s$ of $y / D$ ), to reduce scattering in the curves and make them more visible by means of continuous lines. A case apart is 

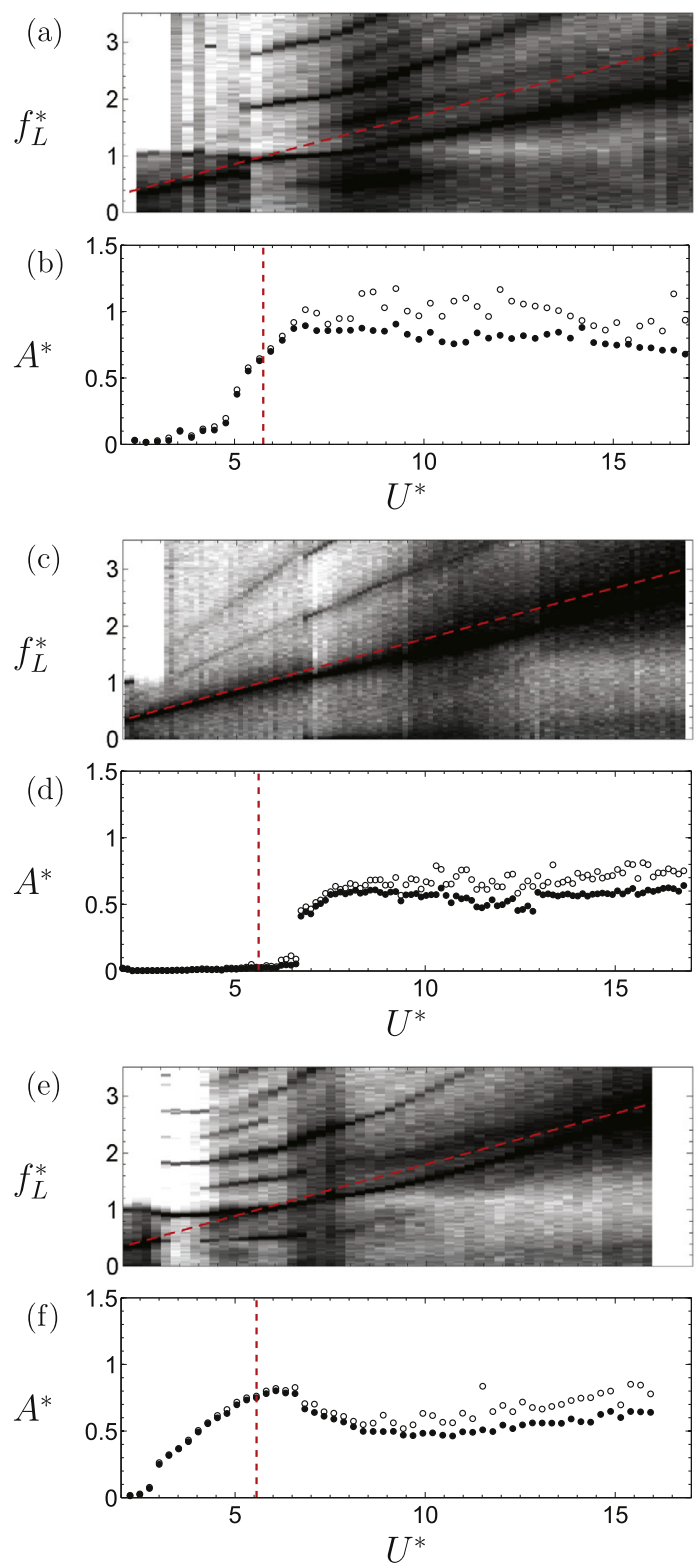

Fig. 14. (a), (c) and (e): Logarithmic-scale reduced frequency power spectrum contours of the lift coefficient $f_{L}^{*}$ and (b), (d) and (f): the normalized amplitude response $A^{*}$ for $\alpha=20^{\circ}, 45^{\circ}$ and $50^{\circ}$, respectively, as a function of $U^{*}$. Open and filled markers indicate $A_{\max }^{*}$ and $A_{10}^{*}$ responses, respectively; the slope dashed lines denote the Strouhal vortex shedding frequency; the vertical dashed lines denote $U^{*}=1 / S t$ in the amplitude response plots.

represented by $\alpha=15^{\circ}$, given that almost null amplitudes were recorded, except around $U^{*}=7.5$ which is characterized by a very chaotic response.

\section{Discussion}

The results presented constitute a somewhat indirect measure of the physical mechanisms that underlie the fluidstructure interactions (FSI) seen in this study. In seeking to interpret such outcomes, it seems likely that there could be added insight and hence significant interest in identifying the transitions between the response types of the rectangular cylinders with the side ratio varying from $S R=1.5$ to $S R=0.67$, through a middle value of $S R=1$. To this end, Fig. 16 shows the comparisons of the amplitude and frequency responses between the present rectangular cylinder with the two side ratios and a square cylinder previously studied by Nemes et al. (2012), where a low-mass-ratio square section was studied in a similar way, i.e. at different angles of attack, and showed, among the other results, the impressive similarity of 

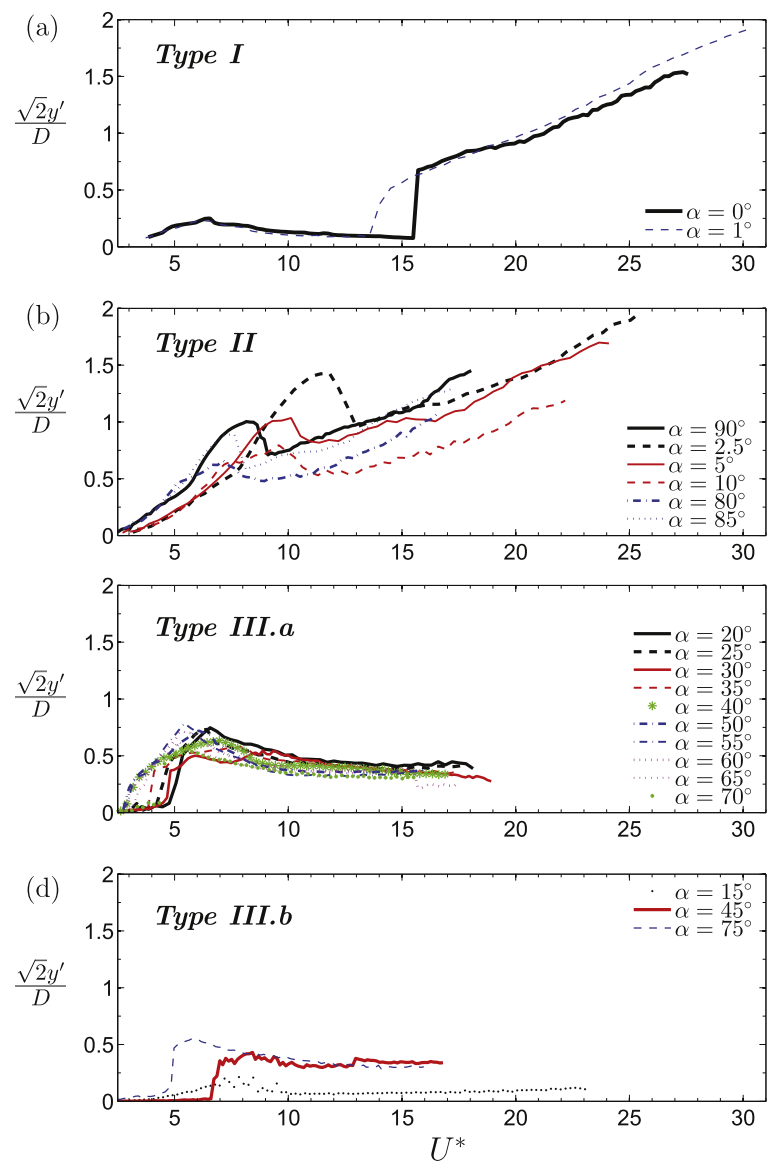

Fig. 15. Equivalent sinusoid nondimensional amplitude response divided in the Types mentioned within the body text.

the response for $\alpha=45^{\circ}$ orientation with that of a circular cylinder. It is worth noting that these two sharp-edged cylinders have comparable mass ratios, that is $m^{*}=2.24$ and $m^{*}=2.2$ for D24/D37 and D25 respectively.

From the amplitude responses, it is apparent that the $S R=1$ case differs more from the $S R=1.5$ case than from the $S R=0.67$. Evidently, the square case is dominated by galloping almost from the beginning of the velocity range (except for a small resonance region at the first two points), whereas both cases of $S R=0.67$ and 1.5 exhibit identifiable VIV (in dark grey) and galloping (in light grey) regions. In particular, a pronounced increase in the amplitude response can be seen in the VIV regions. However, the slopes of the amplitude response show increasing trends in the galloping regions that are comparable for the three cases. It can be seen that the dominant oscillation frequency in the galloping regions tends to become stable at high reduced velocity but it tends to decrease with increasing side ratios, i.e. $f^{*}=0.75,0.64$ and 0.57 for $S R=0.67,1$ and 1.5 , respectively.

It should be noted that while the response of the square cylinder is dominated by galloping, that of the rectangular cylinder with $S R=1.5$ exhibits an apparently different form, with respect to what happens for VIV-galloping interaction in air flow. Theoretically, cross-sections aerodynamically unstable and having very low mass and damping ratio, fall in the case of "asynchronous quenching" (firstly proposed by Minorsky (1947) and recalled in Santosham (1966), see in the Introduction), implying that the "auto-periodic" oscillations are suppressed by the "hetero-periodic" ones, meaning that the vortex-shedding has a stabilizing effect on the body. Otherwise, under the action of the quasi-steady term of forces, it would be aerodynamically prone to follow the galloping instability branch at the reduced onset velocity predicted by $Q S$-theory, which is presently lower than the resonance one. Such a condition is automatically verified in the case of water flows because the QS-galloping onset theoretical velocity is much lower than that required for VIV (Parkinson, 1989).

To illustrate this, the order of magnitude of the velocity ratio $\Lambda=U_{g}^{*} / U_{r}^{*}$ is equal to $0.0028,0.0037$ and 0.0044 respectively for $S R=1.5,0.67$ and 1, respectively, in the present study, i.e. from a very low velocity range. In air flow, the onset of the instability branch corresponds to $U^{*}=U_{r}^{*}$, where the body is driven by the Kármán vortices, acting as an external excitation to the system (e.g. leading-edge vortex (LEV) shedding, caused by the interaction between upper and lower shear layers in the near-wake), then exhibiting linearly growing unrestricted oscillations. 

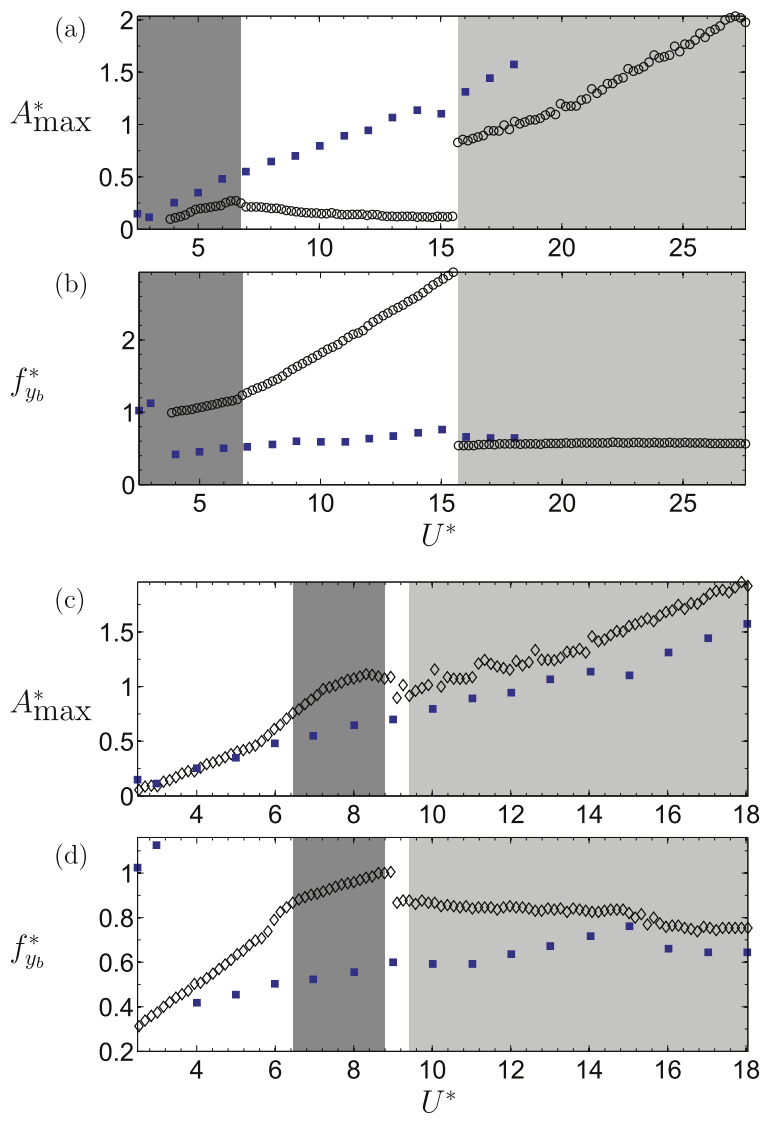

Fig. 16. $A_{\max }^{*}(\mathrm{a})$ and $f_{y_{b}}^{*}(\mathrm{~b})$ response against reduced velocity $U^{*}$, of the D24 rectangular cylinder $(S R=1.5)$. Overlapped, the same kind of representation for the square cylinder D25 (SR = 1, $\mathbf{a}$, blue-coloured online). (c)-(d): D37 configuration $(S R=0.67)$.

Source: D25 data from Nemes et al. (2012).

In cases of low values of mass-damping (i.e. $m^{*}-\zeta$ ) parameter, such as found in air flow, a secondary resonance occurs in the range $0.25 \lesssim U / U_{r} \lesssim 0.5$, the physics of which has been discussed carefully in Mannini et al. (2016). This self-limited secondary form of instability has been recognized to be likely due, for rectangular cylinders with $S R=1.5$ to 2 , to the impinging leading-edge vortices (ILEV) mechanism (Naudascher and Rockwell, 2005). This mechanism arises due to the vortices forming at the leading edge impinging on the trailing edges, which causes alternate pressures on the body sides so to interfere and control the generation of the leading edge vortices (Rockwell and Naudascher, 1979). $S R=1$ and $S R=0.67$ are usually too short in stream-wise length to activate ILEV without experiencing a very large vibration (e.g. at very low Sc values). This means that the secondary resonance does not normally manifest itself, although some examples are present in the literature e.g. Bouclin (1977) and Bokaian et al. (1984), in water flow, for higher mass ratios, and Suda et al. (2016) in air for a $S R=1.18$. For these reasons, the square section response in water flow, here proposed for comparison, is in line with what could be expected according to previous results in the literature. No secondary resonance, amplitudes develop from reduced velocities lower than the resonance one (as reported by Bouclin (1977) for $m^{*} \lesssim 9.79$ and seemingly by Bokaian et al. (1984) for a $m^{*} \simeq 7.17$ ). Even the response of $S R=0.67$ qualitatively and quantitatively corresponds to the one obtained by Bokaian and Geoola (1985), in which, for a $S R=0.5$, two test cases were shown having an $m^{*} \simeq 9.96$ and $m^{*} \simeq 16.9$. It can be seen in figure 8 of Bokaian and Geoola (1985) that unrestricted amplitude developing linearly from rest and presenting an amplified amplitude response around $U_{r}^{*}$ which makes the body oscillate at an amplitude of about the section depth. This could mean the amplitude response does not depend on the mass ratio for this section.

What is quite unusual is the behaviour of the $S R=1.5$ case. The only comparable case is the rectangular cylinder with $S R=1.5$ and $m^{*} \simeq 12.48$ reported in Bokaian and Geoola (1983), and the $S R=2$ and $m^{*} \simeq 11.52$ tested by Bouclin (1977). In Bokaian and Geoola's study, the $S R=1.5$ rectangle cylinder develops only one branch of instability, having its onset at $U_{r}^{*}$ and presenting a clear change in the amplitude response slope at $U^{*} \simeq 25.5$, similar to the present $S R=1.5$ case at $U^{*} \simeq 20$. However, the desynchronization region after the resonance at very low reduced velocities, characterized by slowly decreasing oscillation amplitudes and increasing dominant $f^{*}$ response, is completely missing. On the other hand, Bouclin's rectangular cylinder with $S R=2$, shows a secondary resonance, occurring before $U_{r}^{*}$ and centred at $U_{r}^{*} / 2\left(0.3<U / U_{r}<0.68\right)$. The principal galloping instability develops from here, following the trend of the galloping 
branch of $S R=1.5$. In contrast, the frequency response of Bouclin's $S R=2$ presents different features to the $S R=1.5$ case. Furthermore, it shows no upward jump in the amplitude response and no sharp cut in the frequency response (see respectively Fig. 16(a), (b) around $U^{*} \approx 16$ ) are apparent, though $f^{*}$ reaches a value of about 0.75 at the highest velocities tested (more in line with the value presently given for $S R=0.67$ and 1 ). Hence, the $S R=1.5$ case appears to be highly sensitive to the effect of the mass ratio. This behaviour is similar to the circular cylinder case with studies of Khalak and Williamson (1996) for very low mass ratio in water, compared to a much higher value found in air by Feng (1968).

Here the rectangle response of $S R=1.5$, the different form of interaction and the VIV-galloping plays its role in the sense that the quasi-steady component of forcing term is dominated by the non-stationary part in the lower range of velocity. At a certain value of velocity the negative aerodynamic damping abruptly occurs, in the form of the above described sudden jump upwards in amplitude, making the response curve to switch in the pure quasi-steady galloping branch. The effect of VIV is present in both $S R=0.67$ and 1.5 response curves, though in a different way, given that for both it implies the oscillation amplitudes increase in the lock-in range (Fig. 16). Outside the resonance regions we observe a different behaviour: while for $S R=1.5$, in the desynchronization region, the vortex shedding is able to almost stabilize the body before the violent onset of galloping branch, in the case of $S R=0.67$ the resonance intervenes when the quasi-steady galloping branch has already taken place.

\section{Conclusions}

The effect of angle of attack variation on the flow-induced vibration response of a rectangular cylinder with low side ratio $(S R=1.5)$ and low mass ratio $\left(m^{*}=2.24\right)$ has been investigated experimentally in water flow. Starting from the crosssection aerodynamics, the characteristics in terms of the vibration amplitude and frequency of the hydroelastic system with two orientations, namely $\alpha=0^{\circ}(S R=1.5)$ and $90^{\circ}(S R=0.67)$, have been shown.

Measurements of the Strouhal number revealed that for an angle of attack covering the entire range from $-5^{\circ} \leqslant \alpha \leqslant 105^{\circ}$, the results agree well with previous work. Another key factor, i.e. the lateral force coefficient $C_{\mathrm{Fy}}$, has been experimentally determined for the two main orientations $\left(\alpha=0^{\circ}\right.$ and $\left.90^{\circ}\right)$ both resulted soft-type oscillators with respect to galloping instability. Neglecting any consideration of the effect of turbulence, it can be derived here and also using previous literatures' results, that for $S R=0.67$ (but the same can be extended to $S R=0.5$ case, which is more studied in literature) it exhibits the form of a soft-type oscillator in water flow conditions, whilst being a hard-type oscillator in air flow.

Different response Types have been categorized based on the characteristics of the amplitude and frequency response. Three major response types have been identified, more or less dividing the angle of attack range into three distinct parts, underlying some trends in the evolution of the response curves. Representative cases for each response Type have been analysed in-depth, in particular by the means of a frequency response analysis providing detailed frequency components and corresponding PSD contours over the reduced velocity range investigated. A brief summary of the present findings is given below for the sake of clarity:

- Type I, obtained for the quasi-zero attack angles of the $S R=1.5$ case, exhibits a clear VIV response in the lowest velocities range, though far below the critical velocity, and a galloping branch in the higher velocity range of the response curve. These two different response branches are separated by a desynchronization branch. The most interesting aspect is the sudden jump in oscillation amplitudes at a certain value of reduced velocity, sharply dividing the desynchronization from the galloping branches. The three different branches are clearly delimited and identifiable in the frequency domain.

- Type II, somewhat curiously starts from $\alpha=2.5^{\circ}$, and characterizes near-zero angles for the two side ratio cases $(S R=1.5$ and 0.67$)$; the VIV and desynchronization region are, however, inverted, and the amplitude response curve presents different features respect to Type I.

- Type III collects all the other amplitude response curves that cannot be ascribed to Type I and II. These show mixed and more chaotic properties, for both the amplitude and frequency response domain.

The results for the $S R=1.5$ have been compared with those of a previously-studied square cylinder, and also with rectangular cylinders of comparable $S R$ in previous studies. The comparison between the present rectangle and the square cross-section shows they lack of any form of resonance or desynchronization occurring in the galloping response branch, which linearly develops almost from rest. The results revealed the key-role played by the mass ratio in determining the occurrence of such a form of instability, which would warrant further investigation, perhaps using direct measurement techniques such as particle-image velocimetry.

\section{Acknowledgements}

The authors acknowledge the financial support from Australian Research Council Discovery Project, Australia grants DP150103177, DP150102879, and DP170100275. In addition, T. Massai would like to thank the FLAIR group in the Department of Mechanical and Aerospace Engineering, Monash University for the experimental facilities made available, and also thank Dr. K. W. L. Wong for his valuable contributions to the development of the experimental rotational rig employed in the static measurements. Thanks also to Dr. Claudio Mannini for the personal and scientific support during the experimental, data analysis and interpretation phases. 


\section{References}

Bearman, P., 1967. On vortex street wakes. J. Fluid Mech. 28 (4), 625-641.

Bearman, P., 1984. Vortex shedding from oscillating bluff bodies. Annu. Rev. Fluid Mech. 16, 195-222.

Bearman, P.W., Currie, I.G., 1979. Pressure uctuation measurements on an oscillating circular cylinder. J. Fluid Mech. 91, 661-677.

Bearman, P.W., Davies, M.E., 1977. The ow about oscillating bluff structures. In: Eaton, K.J. (Ed.), Proc. Int. Conf. Wind Eff. Build. Structures, $4^{\text {th }}$. Cambridge Univ. Press., pp. 285-295

Bearman, P., Obasaju, E., 1982. An experimental study of pressure fluctuations on fixed and oscillating square-section cylinders. J. Fluid Mech. 119, $297-321$. Bloor, M., 1964. The transition to turbulence in the wake of a circular cylinder. J. Fluid Mech. 19 (2), 290-304.

Bokaian, A., Geoola, F., 1983. On the cross flow response of cylindrical structures. ICE Proc. 75, 397-418.

Bokaian, A., Geoola, F., 1985. Effects of vortex resonance on nearby galloping instability. J. Eng. Mech. 111 (5), 591-609.

Bokaian, A., Geoola, F., Obasaju, E., 1984. On the cross flow response of cylindrical structures. ICE Proc. 77, 99-101.

Bouclin, D., 1977. Hydroelastic Oscillations of Square Cylinders (Master's thesis), the University of British Columbia.

Den Hartog, J., 1932. Trasmission line vibration due to slit. Trans. EE 51 (4), 1074-1086.

Feng, C., 1968. The Measurement of Vortex Induced Effects in Flow Past Stationary and Oscillating Circular and D-Section Cylinders (Master's thesis), the University of British Columbia.

Gerrard, J.H., 1966. The mechanics of the formation region of vortices behind bluff bodies. J. Fluid Mech. 25 (02), 401-413.

Glauert, H., March 1919. The rotation of an aerofoil about a fixed axis. Tech. Rep. 595, Advisory Committee for Aeronautics.

Hémon, P., 2012. Large galloping oscillations of a square section cylinder in wind tunnel. In: Proceedings of the $10^{\text {Th }}$ International Conference on FlowInduced Vibration (\& Flow-Induced Noise).

Hover, F., Techet, A., Triantafyllou, M., 1998. Forces on oscillating uniform and tapered cylinders in cross flow. J. Fluid Mech. 363, 97-114.

Khalak, A., Williamson, C., 1996. Dynamics of a hydroelastic cylinder with very low mass and damping.. J. Fluids Struct. 10 (5), 455-472.

Mannini, C., Marra, A., Bartoli, G., 2014. VIV-galloping instability of rectangular cylinders: Review and new experiments. J. Wind Eng. Ind. Aerodyn. 132, 109-124.

Mannini, C., Marra, A., Massai, T., Bartoli, G., 2016. Interference of vortex-induced vibration and transverse galloping for a rectangular cylinder. J. Fluids Struct. 66, 403-423.

Massai, T., 2015. On the Interaction Between Vortex-Induced Vibrations and Galloping in Rectangular Cylinders of Low Side Ratio (Ph.D. thesis), University of Florence - TU Braunschweig.

Minorsky, N., 1947. Introduction to non-linear mechanics: Topological methods, analytical methods, non-linear resonance, relaxation oscillations.

Morse, T., Govardhan, R., Williamson, C., 2008. The effect of end conditions on the vortex-induced vibration of cylinders. J. Fluids Struct. 24 (8), 1227-1239.

Nakamura, Y., Tamonari, Y., 1977. Galloping of rectangular prisms in a smooth and in a turbulent flow. J. Sound Vib. 52 (2), 233-241.

Naudascher, E., Rockwell, D., 2005. Flow-Induced Vibrations: An Engineering Guide. Dover Publications.

Nemes, A., Zhao, J., Lo Jacono, D., Sheridan, J., 2012. The interaction between flow-induced vibration mechanisms of a square cylinder with varying angles of attack. J. Fluid Mech. 710, 102-130. http://dx.doi.org/10.1017/jfm.2012.353.

Novak, M., 1972. Galloping oscillations of prismatic structures. J. Eng. Mech. Div. - Proc. ASCE 98 (EM1), 27-46.

Novak, M., Tanaka, H., 1974. Effect of turbulence on galloping instability. J. Eng. Mech. Div. - Proc. ASCE 100 (EM1), 27-47.

Parkinson, G., 1963. Aeroelastic galloping in one degree of freedom. In: 1st Symposium on Wind Effects on Building and Structures - Teddington, UK, Vol. 1. Her Majesty's Stationery Office, London, p. 582.

Parkinson, G., 1989. Phenomena and modelling of flow-induced vibrations of bluff bodies. Prog. Aerosp. Sci. 26 (2), 169-224.

Parkinson, G., Brooks, P., 1961. On the aeroelastic instability of bluff cylinders. Trans. ASME 252-258.

Parkinson, G., Smith, J., 1964. The square prism as an aeroelastic non-linear oscillator. Quart. J. Mech. Appl. Math. 17 (2), 225-239.

Parkinson, G., Wawzonek, M., 1981. Some considerations of combined effects of galloping and vortex resonance. J. Wind Eng. Ind. Aerodyn. 8 (1-2), $135-143$.

Perry, A.E., Chong, M.S., Lim, T.T., 1982. The vortex-shedding process behind two-dimensional bluff bodies. J. Fluid Mech. 116, 77-90.

Rockwell, D., Naudascher, E., 1979. Self-sustained oscillations of impinging free shear layers.. Annu. Rev. Fluid Mech. 11, 67-94.

Roshko, A., 1954. On the development of turbulent wakes from vortex streets. Tech. Rep. 1191, N.A.C.A. (National Advisory Committee for Aeronautics).

Santosham, T., 1966. Force Measurements on Bluff Cylinders and Aeroelastic Galloping of a Rectangular Cylinder (Ph.D. thesis), the University of British Columbia.

Sarpkaya, T., 1979. Vortex-induced oscillations: A selective review. J. Appl. Mech. 46, 241-258.

Sarpkaya, T., 2004. A critical review of the intrinsic nature of vortex-induced vibrations. J. Fluids Struct. 19 (4), 389-447.

Sherry, M., Lo Jacono, D., Sheridan, J., 2010. An experimental investigation of the recirculation zone formed downstream of a forward facing step. J. Wind Eng. Ind. Aerodyn. 98 (12), 888-894.

Shiraishi, N., Matsumoto, M., 1983. On classification of vortex-induced oscillation and its application for bridge structures. J. Wind Eng. Ind. Aerodyn. 14(1-3), 419-430.

Smith, J.D., 1962. An Experimental Study of the Aeroelastic Instability of Rectangular Cylinders (Master's thesis), University of British Columbia.

Suda, K., Matsuda, K., Kato, K., Tamai, Y., 2016. Study on aerodynamic vibrations of a bracing member with a rectangular cross-section of along-spanned truss bridge. In: Proceedings of the $1^{\text {St }}$ International Symposium on Flutter and Its Application - ISFA (Tokyo, Japan).

Sumer, B., Fredsøe, J., 1997. Hydrodynamics Around Cylindrical Structures, first ed.. In: Advanced Series on Ocean Engineering, vol. 12, World Scientific.

Wong, K.W.L., Zhao, J., LoJacono, D., Thompson, M.C., Sheridan, J., 2017. Experimental investigation of flow-induced vibration of a rotating circular cylinder. J. Fluid Mech. 829, 486-511. http://dx.doi.org/10.1017/jfm.2017.540.

Zhao, J., Hourigan, K., Thompson, M.C., 2018a. Flow-induced vibration of d-section cylinders: an afterbody is not essential for vortex induced vibration. J. Fluid Mech. 851, 317-343.

Zhao, J., Leontini, J., Lo Jacono, D., Sheridan, J., 2014a. Fluid-structure interaction of a square cylinder at different angles of attack. J. Fluid Mech. 747, 688-721.

Zhao, J., Leontini, J.S., Lo Jacono, D., Sheridan, J., 2014b. Chaotic vortex induced vibrations. Phys. Fluids 26 (12), 121702.

Zhao, J., Lo Jacono, D., Sheridan, J., Hourigan, K., Thompson, M.C., 2018b. Experimental investigation of in-line flow-induced vibration of a rotating cylinder. J. Fluid Mech. 847, 664-699. 